\title{
Microlensing variability in the gravitationally lensed quasar QSO 2237+0305 三 the Einstein Cross *
}

\section{Spectrophotometric monitoring with the VLT}

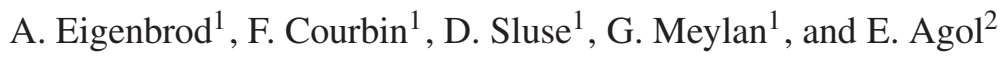 \\ 1 Laboratoire d'Astrophysique, École Polytechnique Fédérale de Lausanne (EPFL), Observatoire de Sauverny, 1290 Versoix, \\ Switzerland \\ 2 Astronomy Department, University of Washington, Box 351580, Seattle, WA 98195, USA
}

Received 18 September 2007 / Accepted 20 December 2007

ABSTRACT

\begin{abstract}
We present the results of the first long-term (2.2 years) spectroscopic monitoring of a gravitationally lensed quasar, namely the Einstein Cross QSO 2237+0305. The goal of this paper is to present the observational facts to be compared in follow-up papers with theoretical models to constrain the inner structure of the source quasar.

We spatially deconvolve deep VLT/FORS1 spectra to accurately separate the spectrum of the lensing galaxy from the spectra of the quasar images. Accurate cross-calibration of the 58 observations at 31-epoch from October 2004 to December 2006 is carried out with non-variable foreground stars observed simultaneously with the quasar. The quasar spectra are further decomposed into a continuum component and several broad emission lines to infer the variations of these spectral components.

We find prominent microlensing events in the quasar images $\mathrm{A}$ and $\mathrm{B}$, while images $\mathrm{C}$ and $\mathrm{D}$ are almost quiescent on a timescale of a few months. The strongest variations are observed in the continuum of image A. Their amplitude is larger in the blue (0.7 mag) than in the red $(0.5 \mathrm{mag})$, consistent with microlensing of an accretion disk. Variations in the intensity and profile of the broad emission lines are also reported, most prominently in the wings of the C III and center of the C IV emission lines. During a strong microlensing episode observed in June 2006 in quasar image A, the broad component of the C III is more highly magnified than the narrow component. In addition, the emission lines with higher ionization potentials are more magnified than the lines with lower ionization potentials, consistent with the results obtained with reverberation mapping. Finally, we find that the $V$-band differential extinction by the lens, between the quasar images, is in the range $0.1-0.3 \mathrm{mag}$.
\end{abstract}

Key words. gravitational lensing - galaxies: quasars: general - galaxies: quasars: emission line quasars: individual: QSO 2237+0305, Einstein Cross

\section{Introduction}

The gravitational lens QSO 2237+0305, also known as "Huchra's lens" or the "Einstein Cross", was discovered by Huchra et al. (1985) during the Center for Astrophysics Redshift Survey. It consists of a $z_{s}=1.695$ quasar gravitationally lensed into four images arranged in a crosslike pattern around the nucleus of a $z_{l}=0.0394$ barred Sab galaxy. The average projected distance of the images from the lens center is $0.9^{\prime \prime}$.

A few years after this discovery, Schneider et al. (1988) and Kent \& Falco (1988) computed the first simple models of the system, leading to the conclusion that this system was very promising to study microlensing. Indeed, the predicted time delays between the four quasar images are of the order of a day (Rix et al. 1992; Wambsganss \& Paczyński 1994), meaning that intrinsic variability of the quasar can easily be distinguished from microlensing events. In addition, the particularly small redshift of the lensing galaxy implies large tangential velocities for the microlenses. Furthermore the quasar images form right in the bulge of the lens where the stellar density is the highest. The combination of these properties makes microlensing events very

* Based on observations made with the ESO-VLT Unit Telescope \# 2 Kueyen (Cerro Paranal, Chile; Proposals 073.B-0243(A\&B), 074.B-0270(A), 075.B-0350(A), 076.B-0197(A), 177.B-0615(A\&B), PI: F. Courbin). likely in the Einstein Cross and very rapid, with timescales of a few weeks to a few months. Indeed, Irwin et al. (1989) reported significant brightness variations of the brightest quasar image A, which they interpreted as the first detection ever of microlensing in the images of a multiply-imaged quasar.

Since then, microlensing events have been observed in several other gravitationally lensed quasars, and are expected to occur in virtually any quadruply lensed quasar (Witt et al. 1995). Probably the most compelling examples of microlensing light curves are given by the Optical Gravitational Lensing Experiment (OGLE) (Woźniak et al. 2000a; Udalski et al. 2006). Started in 1997, this project monitors regularly the four quasar images of QSO 2237+0305, showing continuous microlensinginduced variations in the light curves.

Most of the quasar microlensing studies so far are based exclusively on broad-band photometric monitoring (e.g. Woźniak et al. 2000b; Schechter et al. 2003; Colley \& Schild 2003). These observations, even though dominated by variations of the continuum, make it very difficult to disentangle variations in the continuum from variations in the broad emission lines (BELs). Both types of regions are affected by microlensing, but in different ways depending on their size.

Microlensing of an extended source can occur when its size is smaller than or comparable to the Einstein radius of a star, i.e. of the order of $10^{17} \mathrm{~cm}$ or $10^{-1} \mathrm{pc}$ in the case of the Einstein 
Cross (Nemiroff 1988; Schneider \& Wambsganss 1990). From reverberation mapping, the broad line region (BLR) was long estimated to be larger than this, of the order of $10^{18} \mathrm{~cm}$ or $1 \mathrm{pc}$, hence leaving little room for BEL microlensing. However, more recent reverberation mapping studies revise this downwards, to $10^{16} \mathrm{~cm}$ (Wandel et al. 1999; Kaspi et al. 2000), which is also consistent with the disk-wind model of Murray et al. (1995). Inspired by these numbers, Abajas et al. (2002) and Lewis \& Ibata (2004) investigated BEL microlensing in further detail and computed possible line profile variations for various BLR models.

Observations of significant continuum and BEL microlensing have been reported in a number of systems (QSO 2237+0305, Filippenko 1989; Lewis et al. 1998; Wayth et al. 2005; HE 2149-2745, Burud et al. 2002a; HE 0435-1223, Wisotzki et al. 2003; H 1413+117, Chartas et al. 2004; SDSS J1004+4112, Richards et al. 2004; HE 1104-1805, Gómez-Álvarez 2004; HE 0047-1756, Wisotzki et al. 2004; SDSS J0924+0219, Eigenbrod et al. 2006a; Keeton et al. 2006; and RXJ 1131-1231, Sluse et al. 2007). These first observational indications of microlensing can be turned into a powerful tool to probe the inner parts of quasars, provided regular spectroscopic data can be obtained. Several theoretical studies show how multiwavelength light curves can constrain the energy profile of the quasar accretion disk and also the absolute sizes of the line-emitting regions (e.g., Agol \& Krolik 1999; Mineshige \& Yonehara 1999; Abajas et al. 2002; Kochanek 2004).

In this paper we present the results of the first long-term spectrophotometric monitoring of the Einstein Cross. The spectral variations of the four quasar images are followed under sub-arcsecond seeing conditions with the Very Large Telescope (VLT) of the European Southern Observatory for more than two years, from October 2004 to December 2006, with a mean temporal sampling of about one point every second week. This first paper describes the observations, the method used to separate the quasar spectra from that of the lensing galaxy, and the main observational results. Simple considerations of the properties of microlensing caustics and of the geometry of the central parts of the quasar already allow us to infer interesting constraints on the quasar energy profile in the Einstein Cross.

The full analysis of our monitoring data, still being acquired at the VLT, requires detailed microlensing simulations coupled with quasar models and will be the subject of future papers.

\section{Observations}

We acquired our observations with the FOcal Reducer and low dispersion Spectrograph (FORS1), mounted on Kueyen, the Unit Telescope \# 2 of the ESO Very Large Telescope (VLT) located at Cerro Paranal (Chile). We performed our observations in the multi-object spectroscopy (MOS) mode. This strategy allowed us to get simultaneous observations of the main target and of four stars used as reference point-spread functions (PSFs). These stars were used to spatially deconvolve the spectra, as well as to perform accurate flux calibration of the target spectra from one epoch to another. We chose these stars to be located as close as possible to QSO 2237+0305 and to have similar apparent magnitudes as the quasar images. The PSF stars \# 1, 2, 3, and 4 have $R$-band magnitudes of $17.5,17.0,15.5$, and 17.5 mag, respectively. Figure 1 shows the field of view of our observations.

We used the high-resolution collimator of FORS 1 to achieve the best possible spatial sampling of the data, i.e. $0.1^{\prime \prime}$ per pixel. With this resolution, we observed a maximum of 8 objects simultaneously over a field of view of $3.4^{\prime} \times 3.4^{\prime}$. One slit was

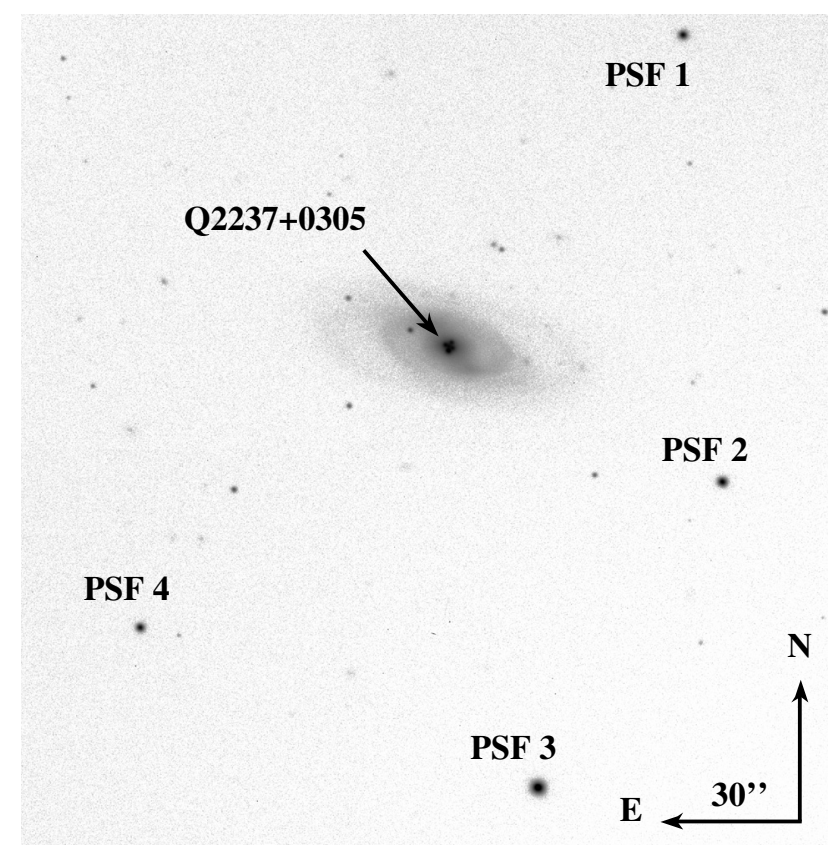

Fig. 1. VLT/FORS1 field of view showing the lensed quasar QSO 2237+0305, along with the four PSF stars used to spatially deconvolve the spectra. These stars are also used to cross-calibrate the observations in flux from one epoch to another and to minimize the effect of sky transparency.

aligned along two of the quasar images and four slits were centered on foreground PSF stars. We placed the remaining slits on empty sky regions and used them to carry out sky subtraction of the quasar data.

Two masks were designed to observe the two pairs of quasar images. The PSF stars in both masks were the same. Figure 2 shows the slit positioning with respect to our target. The first mask was aligned on quasar images $\mathrm{A}$ and $\mathrm{D}$, while the second was aligned on images $\mathrm{B}$ and $\mathrm{C}$. The masks were rotated to position angles that avoid clipping of any quasar image. This is mandatory to carry out spatial deconvolution of the spectra.

Our observing sequences consisted of a short acquisition image, an "image-through-slit" check, followed by a consecutive deep spectroscopic exposure. All individual exposures were $1620 \mathrm{~s}$ long. We list the journal of our observations in Table 1. The mean seeing during the three observing seasons was $0.8^{\prime \prime}$. We chose a slit width of $0.7^{\prime \prime}$, approximately matching the seeing and much smaller than the mean separation of $1.4^{\prime \prime}$ between the quasar images. This is mandatory to avoid contamination of an image by the others.

We used the G300V grism in combination with the GG375 order sorting filter. For our slit width, the spectral resolution was $\Delta \lambda=15 \AA$, as measured from the FWHM of night-sky emission lines, and the resolving power was $R=\lambda / \Delta \lambda \simeq 400$ at the central wavelength $\lambda=5900 \AA$. The useful wavelength range was $3900<\lambda<8200 \AA$ with a scale of $2.69 \AA$ per pixel in the spectral direction. This configuration favors spectral coverage rather than spectral resolution, allowing us to follow the continuum over a broad spectral range, starting with the very blue portion of the optical spectrum. Even so and in spite of $R=400$, a detailed profile of the BEL is still accessible.

We also observed spectrophotometric standard stars (GD 108, HD 49798, LTT 377, LTT 1020, LTT 1788, and LTT 7987) to remove the response of the telescope, CCD, and 


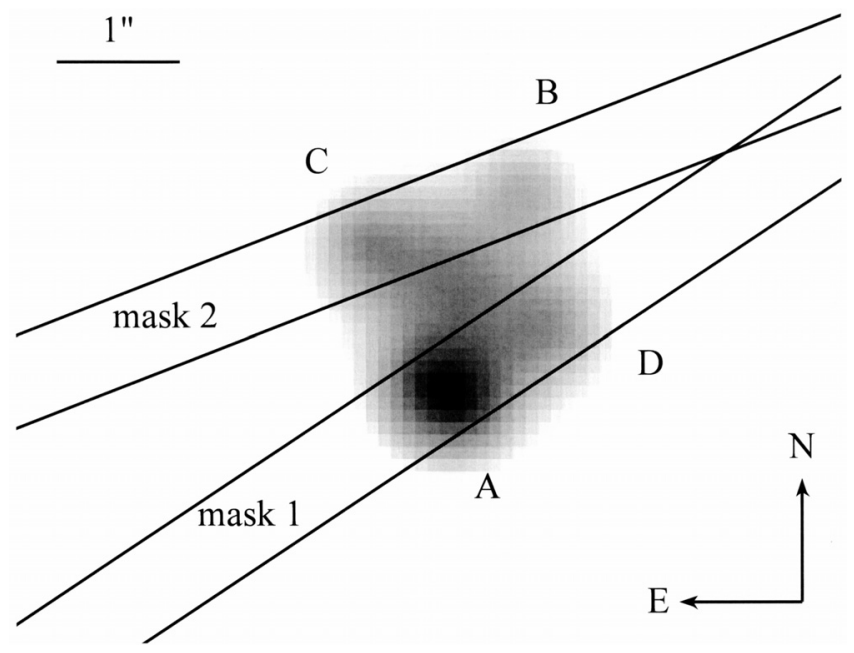

Fig. 2. FORS1 $R$-band acquisition image of QSO 2237+0305 taken on epoch \# 12 (12-09-2005). The slits used in the two masks are shown. Their width is $0.7^{\prime \prime}$. The Position Angle (PA) of mask 1 is $\mathrm{PA}=-56.5^{\circ}$ and that of mask 2 is $\mathrm{PA}=-68.5^{\circ}$.

grism. We did the relative calibration between the epochs using the PSF stars (see for more details Eigenbrod et al. 2006a).

Finally, it is worth emphasizing that all our VLT data used in the present paper were obtained in service mode, without which this project would have been impossible.

\section{Data analysis}

\subsection{Reduction}

The data reduction followed the same procedure described in detail in Eigenbrod et al. (2006b). We carried out the standard bias subtraction and flat field correction of the spectra using IRAF $^{1}$. We obtained the wavelength calibration from the spectrum of helium-argon lamps. All spectra, for the object and for the PSF stars were calibrated in two dimensions.

Only one single exposure was taken per mask and per epoch. For this reason, the usual cosmic-ray rejection scheme applied to multiple images could not be applied. Instead, we used the L. A. Cosmic algorithm (van Dokkum 2001), that can handle single images. We visually inspected the cosmic-ray corrected images to check that no data pixel was affected by the process, especially in the emission lines and in the data with the best seeing.

We removed the sky background in a different way in the spectra of the PSF stars and in those of the gravitational lens. For the PSF stars, which are small compared with the slit length $\left(19^{\prime \prime}\right)$, we used the IRAF task background. This task fits a second order Chebyshev polynomial in the spatial direction to the areas of the spectrum that are not illuminated by the object, and subtracts it from the data. As the lensing galaxy in QSO $2237+0305$ is larger than the slit length, this procedure is not applicable. Instead, we used the slits positioned on empty sky regions of the FORS1 field of view, and located next to the gravitational lens. The sky was fitted to these slits and removed from the slit containing the images of QSO 2237+0305.

${ }^{1}$ IRAF is distributed by the National Optical Astronomy Observatories, which are operated by the Association of Universities for Research in Astronomy, Inc., under cooperative agreement with the National Science Foundation.
Table 1. Journal of the observations taken on 31 epochs.

\begin{tabular}{|c|c|c|c|c|c|}
\hline 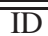 & Civil date & 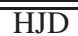 & "Mask & Seeing ["] & "Airmass \\
\hline 1 & $13-10-2004$ & 3292 & 1 & 0.86 & 1.204 \\
\hline 1 & $14-10-2004$ & 3293 & 2 & 0.87 & 1.221 \\
\hline 2 & $14-11-2004$ & 3324 & 1 & 0.75 & 1.184 \\
\hline 2 & $14-11-2004$ & 3324 & 2 & 0.68 & 1.305 \\
\hline 3 & $01-12-2004$ & 3341 & 1 & 0.88 & 1.355 \\
\hline 3 & $01-12-2004$ & 3341 & 2 & 0.94 & 1.609 \\
\hline 4 & $15-12-2004$ & 3355 & 1 & 0.99 & 1.712 \\
\hline 4 & $16-12-2004$ & 3356 & 2 & 0.90 & 1.817 \\
\hline 5 & $11-05-2005$ & 3502 & 1 & 0.87 & 1.568 \\
\hline 5 & $12-05-2005$ & 3503 & 2 & 0.51 & 1.389 \\
\hline 6 & $01-06-2005$ & 3523 & 1 & 0.63 & 1.342 \\
\hline 6 & $01-06-2005$ & 3523 & 2 & 0.64 & 1.224 \\
\hline 7 & $01-07-2005$ & 3553 & 1 & 0.57 & 1.153 \\
\hline 8 & $14-07-2005$ & 3566 & 1 & 0.89 & 1.620 \\
\hline 9 & $06-08-2005$ & 3589 & 1 & 0.51 & 1.135 \\
\hline 9 & $06-08-2005$ & 3589 & 2 & 0.61 & 1.173 \\
\hline 10 & $15-08-2005$ & 3598 & 1 & 0.86 & 1.140 \\
\hline 11 & $25-08-2005$ & 3608 & 1 & 0.49 & 1.261 \\
\hline 11 & $25-08-2005$ & 3608 & 2 & 0.54 & 1.461 \\
\hline 12 & $12-09-2005$ & 3626 & 1 & 0.70 & 1.535 \\
\hline 12 & $12-09-2005$ & 3626 & 2 & 0.69 & 1.341 \\
\hline 13 & $27-09-2005$ & 3641 & 1 & 0.92 & 1.480 \\
\hline 13 & $27-09-2005$ & 3641 & 2 & 0.73 & 1.281 \\
\hline 14 & $01-10-2005$ & 3645 & 1 & 0.78 & 1.281 \\
\hline 14 & $01-10-2005$ & 3645 & 2 & 0.87 & 1.156 \\
\hline 15 & $11-10-2005$ & 3655 & 1 & 0.57 & 1.140 \\
\hline 15 & $11-10-2005$ & 3655 & 2 & 0.66 & 1.134 \\
\hline 16 & $21-10-2005$ & 3665 & 1 & 0.70 & 1.215 \\
\hline 16 & $21-10-2005$ & 3665 & 2 & 0.74 & 1.156 \\
\hline 17 & $11-11-2005$ & 3686 & 1 & 0.90 & 1.137 \\
\hline 17 & $11-11-2005$ & 3686 & 2 & 0.90 & 1.185 \\
\hline 18 & $24-11-2005$ & 3699 & 1 & 0.78 & 1.265 \\
\hline 18 & $24-11-2005$ & 3699 & 2 & 0.90 & 1.443 \\
\hline 19 & $06-12-2005$ & 3711 & 1 & 1.10 & 1.720 \\
\hline 19 & $06-12-2005$ & 3711 & 2 & 1.09 & 1.445 \\
\hline 20 & $24-05-2006$ & 3880 & 1 & 0.87 & 1.709 \\
\hline 20 & $24-05-2006$ & 3880 & 2 & 0.90 & 1.443 \\
\hline 21 & $16-06-2006$ & 3903 & 1 & 0.66 & 1.213 \\
\hline 21 & $16-06-2006$ & 3903 & 2 & 0.51 & 1.155 \\
\hline 22 & $20-06-2006$ & 3907 & 1 & 0.64 & 1.286 \\
\hline 22 & $20-06-2006$ & 3907 & 2 & 0.58 & 1.189 \\
\hline 23 & $27-06-2006$ & 3914 & 1 & 0.41 & 1.145 \\
\hline 23 & $27-06-2006$ & 3914 & 2 & 0.50 & 1.133 \\
\hline 24 & $27-07-2006$ & 3944 & 1 & 0.74 & 1.316 \\
\hline 24 & $27-07-2006$ & 3944 & 2 & 0.76 & 1.204 \\
\hline 25 & $03-08-2006$ & 3951 & 1 & 0.73 & 1.246 \\
\hline 25 & $03-08-2006$ & 3951 & 2 & 0.65 & 1.169 \\
\hline 26 & $13-10-2006$ & 4022 & 1 & 0.59 & 1.176 \\
\hline 26 & $13-10-2006$ & 4022 & 2 & 0.52 & 1.300 \\
\hline 27 & $28-10-2006$ & 4037 & 1 & 0.57 & 1.148 \\
\hline 27 & $28-10-2006$ & 4037 & 2 & 0.53 & 1.138 \\
\hline 28 & $10-11-2006$ & 4050 & 1 & 0.89 & 1.515 \\
\hline 28 & $10-11-2006$ & 4050 & 2 & 0.88 & 1.323 \\
\hline 29 & $27-11-2006$ & 4067 & 1 & 0.87 & 1.255 \\
\hline 29 & $27-11-2006$ & 4067 & 2 & 0.92 & 1.391 \\
\hline 30 & $19-12-2006$ & 4089 & 2 & 1.04 & 2.125 \\
\hline 31 & $22-12-2006$ & 4092 & 1 & 0.80 & 2.018 \\
\hline 31 & $23-12-2006$ & 4093 & 2 & 0.76 & 2.248 \\
\hline
\end{tabular}

\subsection{Flux cross-calibration}

Once the cosmic rays and sky background were removed, we applied a flux cross-calibration of the spectra as described by Eigenbrod et al. (2006a), using the four PSF stars. The spectra 


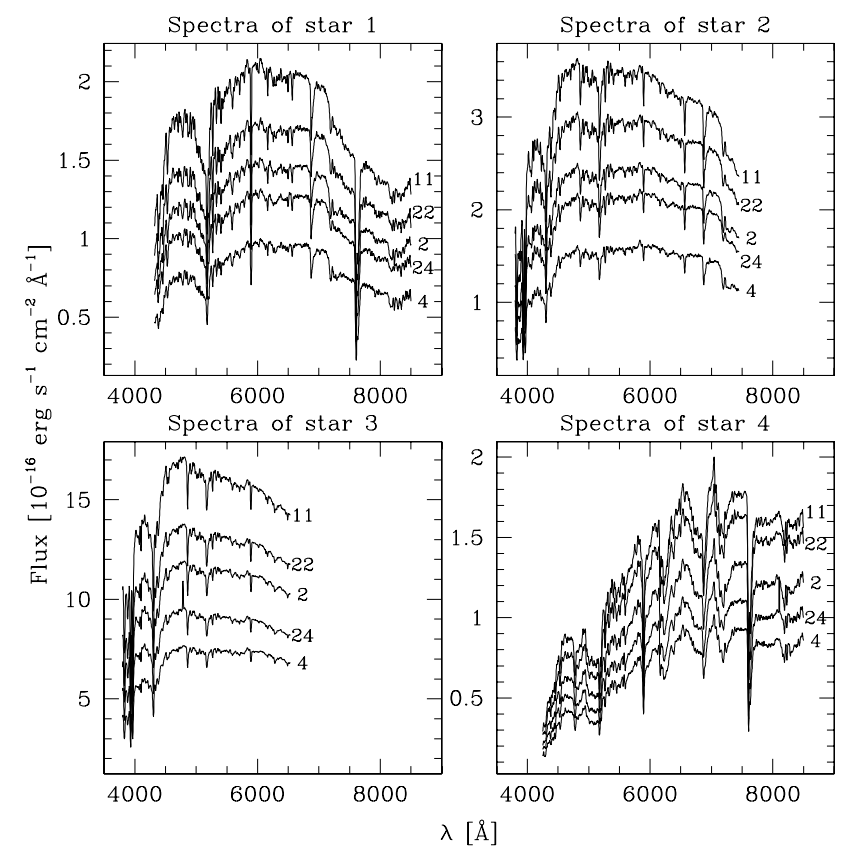

Fig. 3. Spectra of the four PSF stars. The spectra in each panel correspond to different observing epochs, chosen to span the full length of the monitoring. The IDs of the observing epochs, as given in Table 1, are indicated. The differences in flux are mainly due to the presence of thin clouds. The purpose of using these stars as flux cross-calibrators is precisely to eliminate these differences, both in intensity and shape.

of these stars are shown in Fig. 3 for five different observing epochs. Our observations show that these stars are non variable.

We created a ratio spectrum for each star, i.e. we divided the spectrum of the star by the spectrum of the same star for a chosen reference exposure. We chose epoch \# 2 (14-11-2004) as our reference exposure because of the excellent weather conditions at this particular epoch for both seeing and sky transparency. The computation of these flux ratios was done for all four stars in each exposure and we checked the compatibility of the response curves derived with the four different stars are compatible (see Fig. 4). If not, we rejected one or a maximum of two of the PSF stars. This can happen in some exceptional cases, e.g. when the alignment between the star and the slit is not optimal and generates a color gradient in the spectrum of the misaligned object. Aside from this instrumental effect, the observations show no trace of intrinsic variability of the PSF stars.

After checking that the correction spectra obtained for the four stars were very similar, we computed their mean, which we took as the correction to be applied to the gravitational lens. The high stability of the corrections across the field demonstrates that all residual chromatic slit losses due to the atmospheric refraction are fully corrected. This correction is eased by the fact that: (1) the position angle of the masks is the same for the quasar images and for the PSF stars (i.e. the PSF clipping is the same for the target and the reference stars); (2) we avoid observations at large airmasses (i.e. never larger than 2.5); and (3) the atmospheric refraction corrector on FORS1 is very efficient.

\subsection{Deconvolution}

The lensing galaxy in QSO $2237+0305$ is bright. Its central parts have a surface brightness of approximately $18 \mathrm{mag} / \operatorname{arcsec}^{2}$ in the $R$ band, which is comparable to the quasar images. Hence

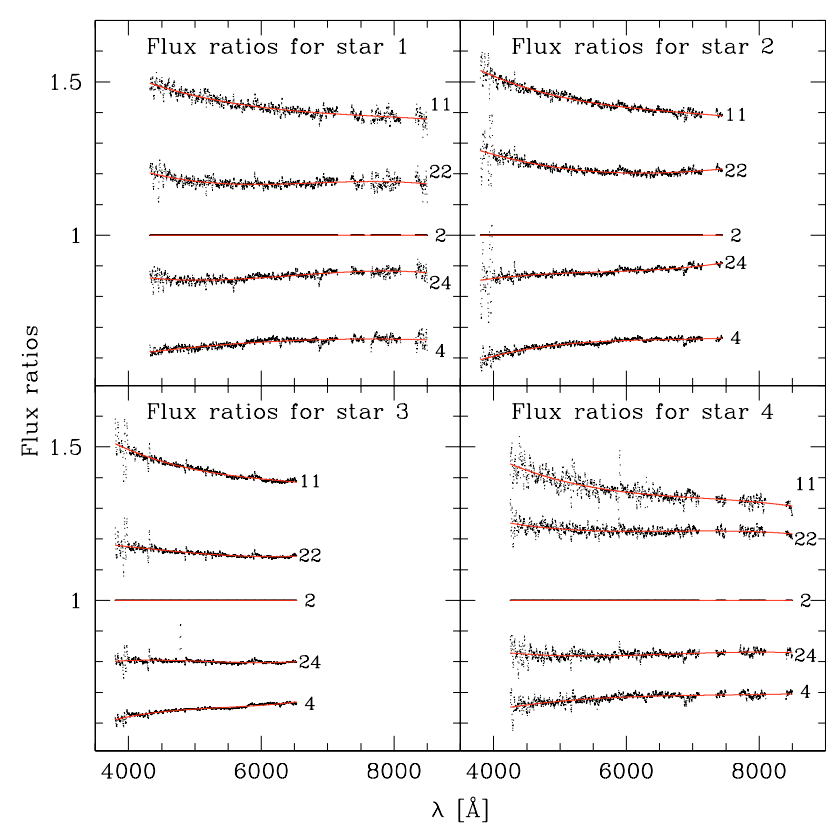

Fig. 4. Flux correction for different epochs with respect to the reference epoch \# 2 (14-11-2004). Each panel corresponds to one of the 4 PSF stars visible in Fig. 3. In each panel, the dotted line shows the ratio of the spectrum of one PSF star taken at a given epoch and the spectrum of the same star taken at the reference epoch \# 2. The curves are polynomials fitting the data. Importantly, the correction derived at a given epoch is about the same for the four stars. The mean of these four curves is used to correct the spectra of the Einstein Cross images. The small parts of the spectra with strong atmospheric absorption are masked. The different spectral ranges are due to different clippings of the spectra by the edges of the CCD.

studying microlensing variations of the quasar images requires very accurate deblending.

In order to carry out this challenging task, we used the spectral version of the MCS deconvolution algorithm (Magain et al. 1998; Courbin et al. 2000), which uses the spatial information contained in the spectra of the PSF stars. The algorithm sharpens the spectra in the spatial direction, and also decomposes them into a "point-source channel" containing the spectra of the two quasar images, and an "extended channel" containing the spectrum of everything in the image that is not a point source, in this case, the spectrum of the lensing galaxy. In Fig. 5, we illustrate the process and the different outputs. In Fig. 7, we show how similar the spectra of the lensing galaxy are, extracted either from two different masks or from data taken at different epochs, hence illustrating the robustness of the deconvolution technique. In Fig. 8, we give an example of decomposition of the data into the quasar and lens spectra after integrating along the spatial direction. The lensing galaxy spectrum shows no trace of the residual quasar BELs. Even when the contrast between the quasar and the galaxy is particularly large, the decomposition is accurate. For example, the $\mathrm{CaII} \mathrm{H}+\mathrm{K}$ doublet in the lens spectrum is well visible, in spite of the presence of the strong quasar $\mathrm{C}$ IV emission in the same wavelength range.

\subsection{Cross-check with the OGLE-III light curves}

After reduction and spatial deconvolution, we obtained the extracted spectra of quasar images A and D on 30 different epochs, 


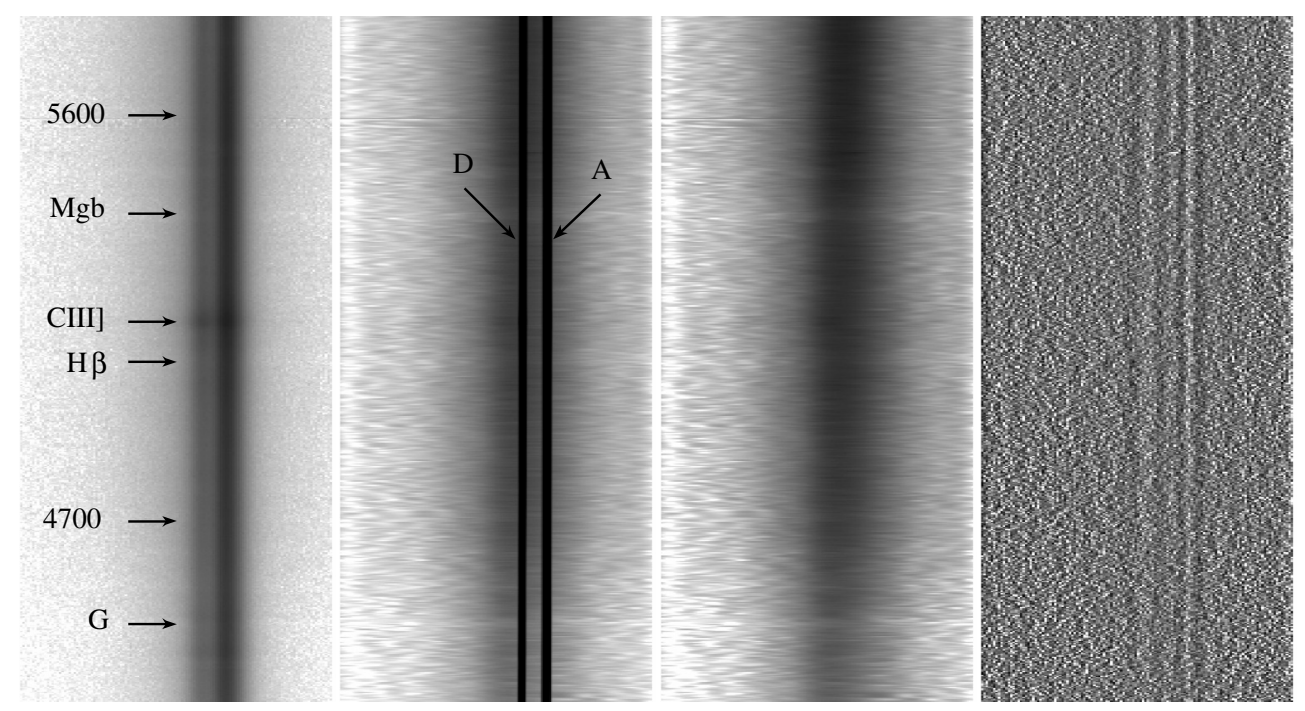

Fig. 5. Left: portion of the VLT 2D-spectrum of quasar images D and A, taken on epoch \# 25 (03-08-2006), on which are indicated the main spectral features of either the quasars or the lens. Center left: spatially deconvolved spectrum. The two quasar images are very well separated. Center right: spectrum of the lensing galaxy alone. Right: residual map of the deconvolution after subtraction of the quasar and lens spectra. Note that the residuals are displayed with much narrower cuts than those used in the other panels. The darkest and brightest pixels correspond to $-3 \sigma$ and $+3 \sigma$ respectively. No significant residuals of the spectral features are visible.

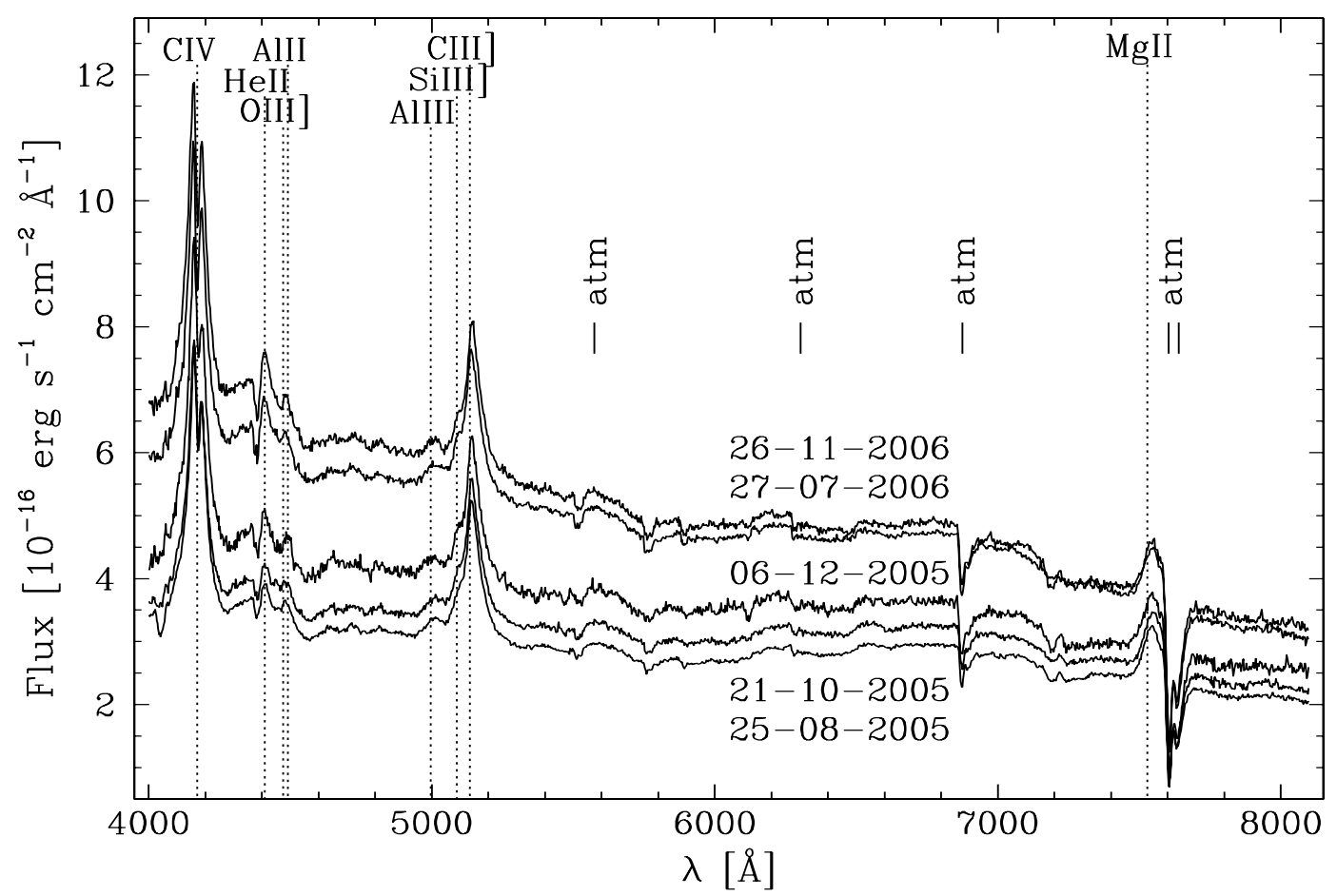

Fig. 6. Deconvolved and extracted spectra of quasar image A for five observing epochs. Chromatic variations in the spectra are conspicuous with the blue part of the spectra being more magnified than the red part.

and of $\mathrm{B}$ and $\mathrm{C}$ on 28 different epochs. Several extracted spectra of image A are shown in Fig. 6. As a sanity check, we compared our results with the OGLE-III photometric monitoring of QSO 2237+0305 (Udalski et al. 2006). We integrated our quasar spectra in the corresponding $V$-band to estimate, from the spectra, the photometric light curves as if they were obtained from imaging. In Fig. 9, we compare our magnitude estimates with the actual OGLE-III measurements. The overall agreement is very good for images $\mathrm{A}, \mathrm{B}$, and $\mathrm{C}$. For image $\mathrm{D}$, we have to shift the OGLE-III light curve by -0.5 mag with respect to the published values. Interestingly, this shift is not needed when we compare our results with the previous OGLE data from the provisional calibration presented in the years 2004-2006. The previous OGLE data also agreed with the photometry of Koptelova et al. (2005). This changed when Udalski et al. (2006) reviewed their calibration and gave image $\mathrm{D}$ a larger magnitude of approximately 0.5 mag. They stated that the steep rise of brightness of image D at the end of the 2000 OGLE-II season leaded to an 


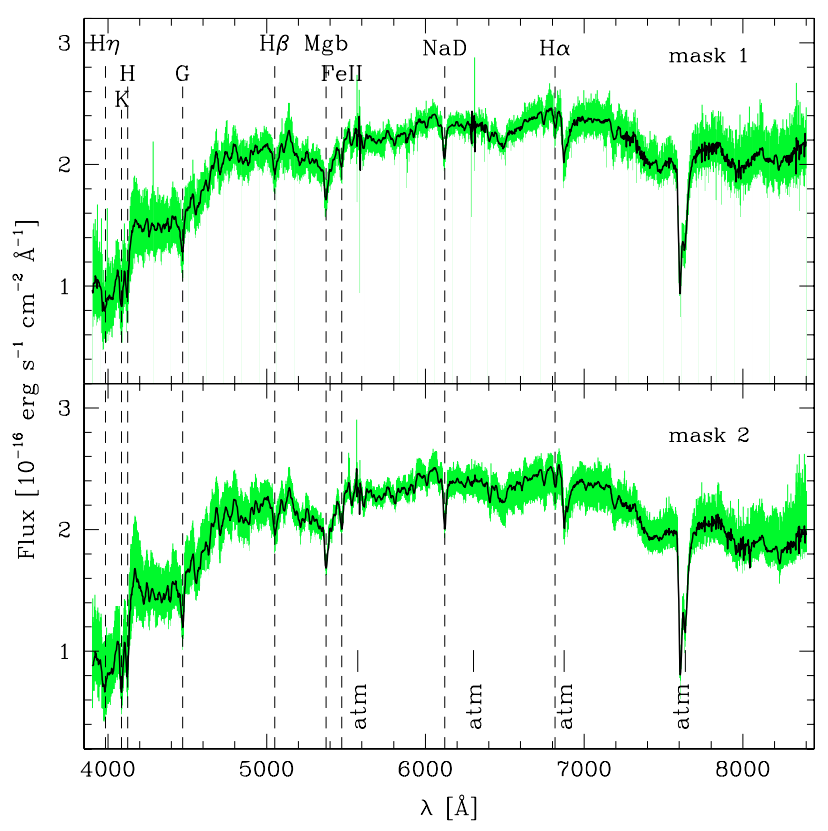

Fig. 7. Deconvolved and extracted 1D-spectra of the lensing galaxy. The two panels correspond to the two MOS masks. The shaded areas are the envelopes containing all the spectra of the lens obtained with the corresponding mask. The thick black lines are the means. Note the small scatter between the two spectra.

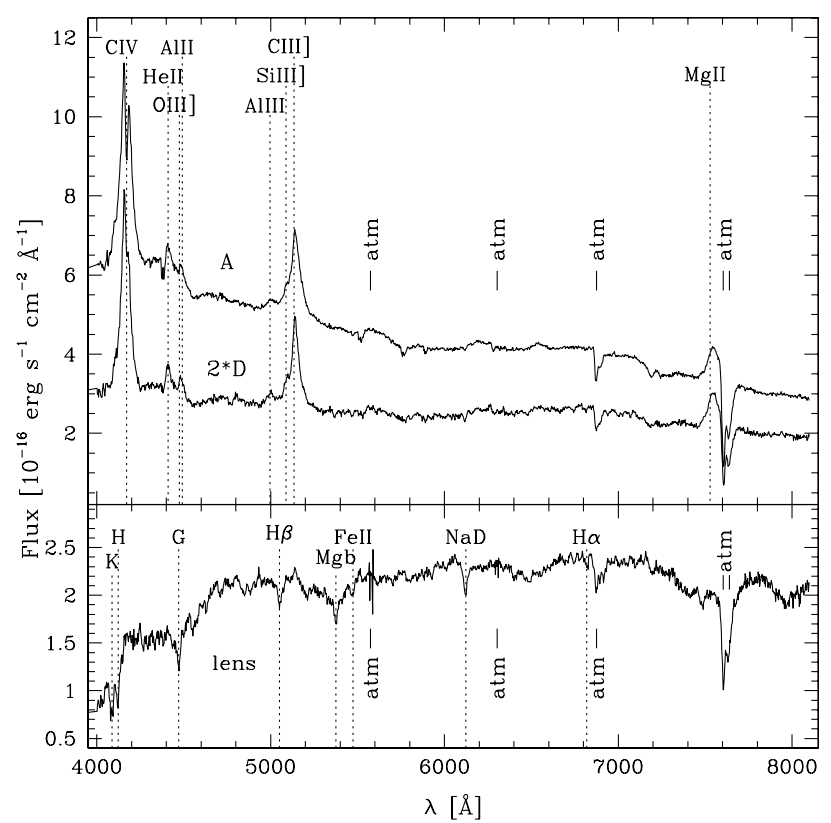

Fig. 8. Example of a spectral decomposition. The top panel shows the two extracted spectra for the images A and D for the observations taken on epoch \# 25 (03-08-2006) with mask 1 . The extracted spectrum of the lensing galaxy, in the bottom panel, shows no trace of contamination by the quasar BELs. For clarit D by a factor of two.

overestimate of the extrapolated magnitude for the beginning of the 2001 OGLE-III season. But this is now discrepant with the photometry of Koptelova et al. (2005). We think that the new extrapolation of the light curve of image D from the end of season 2000 to the beginning of season 2001 might be uncertain,

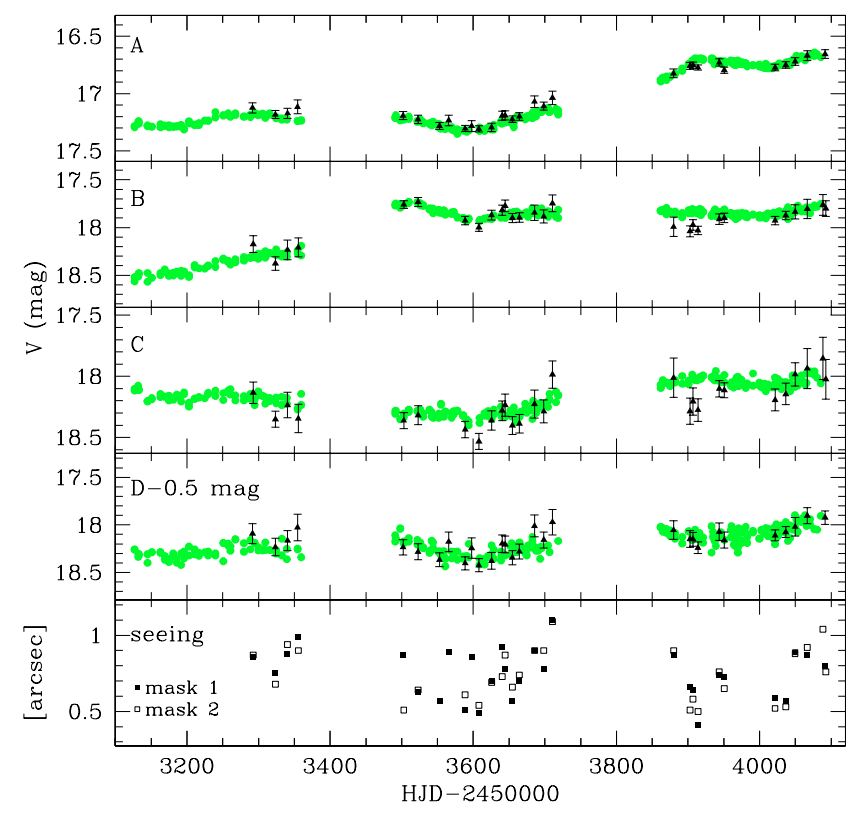

Fig. 9. OGLE-III light curves (Udalski et al. 2006) of all four quasar images from April 2004 to December 2006 (dots), compared with the photometry derived by integrating our VLT spectra through the OGLE $V$-band (dark triangles). The 1-sigma error bars correspond to the photon noise in the spectrum. We shift the OGLE-III light curve of image D by -0.5 mag with respect to the published values. The bottom panel displays the seeing values for each observations.

leading to the observed shift between our data and the OGLE-III light curve of image D. However, aside from this shift, the agreement between the OGLE photometry and our integrated VLT spectra is also very good for image D.

\section{Multi-component decomposition}

Different emission features are known to be produced in regions of different characteristic sizes. As microlensing magnification varies on short spatial scales, sources of different sizes are magnified by differing amounts (e.g. Wambsganss et al. 1990). Emission features from smaller regions of the source are more highly variable due to microlensing than features emitted in more extended regions. In order to study the variation of each spectral feature independently, we need to decompose the spectra into their individual components.

\subsection{Method}

In our analysis of the 1-D spectra of the four quasar images, we follow the multi-component decomposition (MCD) approach (Wills et al. 1985; Dietrich et al. 2003) implemented in Sluse et al. (2007). This method is applied to the rest-frame spectra, assuming they are the superposition of (1) a power law continuum, (2) a pseudo-continuum due to the merging of Fe II and Fe III emission blends, and (3) an emission spectrum due to the other individual BELs. We consider the following emission lines : C IV $\lambda 1549$, He II $\lambda 1640$, O III $\lambda 1664$, Al II $\lambda 1671$, Al II $\lambda 1857$, Si III $\lambda 1892$, C III $\lambda 1909$, and Mg II $\lambda 2798$. All these features are fitted simultaneously to the data using a standard least-square minimization with a Levenberg-Marquardt based algorithm adapted from the Numerical Recipes (Press et al. 1986). 


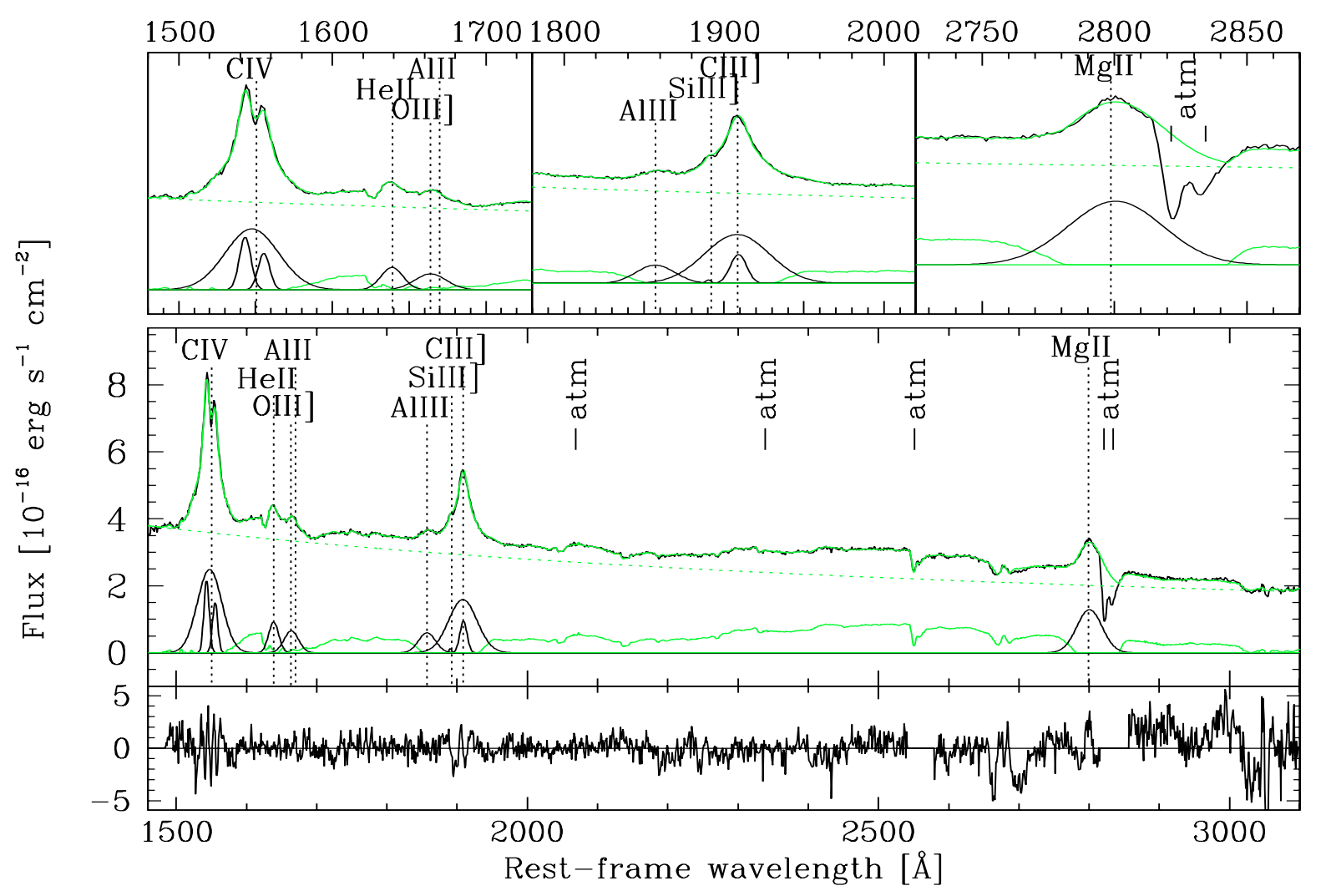

Fig. 10. Multi-component decomposition of the spectrum of the brightest quasar image, A, taken on epoch \# 2 (14-11-2004). The upper panels show the detailed spectral decomposition of the BELs, while the middle panel displays the entire spectrum. The continuum is indicated as a dotted curve. The Gaussian lines and iron pseudo-continuum templates are shown below the spectrum. The bottom panel is the residual for each pixel normalized by the photon noise per pixel (i.e. the $y$-axis is the residual flux in units of $\sigma$ ).

In the first step, we identify the underlying nonstellar powerlaw continuum from spectral windows that are free (or almost free) of contributions from the other components, namely the iron pseudo-continuum and the BELs. We use the windows $1680 \leq \lambda \leq 1710 \AA$ and $3020 \leq \lambda \leq 3080 \AA$. After visual inspection of the iron templates by Vestergaard et al. (2001), we do not expect significant iron emission in these windows.

We characterize the spectral continuum (measured in the restframe) with a power law $f_{v} \propto v^{\alpha_{v}}$, which translates in wavelength to $f_{\lambda} \propto \lambda^{\alpha_{\lambda}}$ with the relation $\alpha_{v}=-\left(2+\alpha_{\lambda}\right)$, i.e.

$f_{\lambda}=f_{0}\left(\frac{\lambda}{\lambda_{0}}\right)^{\alpha_{\lambda}}=f_{0}\left(\frac{\lambda}{\lambda_{0}}\right)^{-\left(2+\alpha_{v}\right)}$

where $\lambda_{0}=2000 \AA$ and where $\alpha_{v}$ is the commonly used canonical power index.

Next, we fit the BELs with Gaussian profiles. We consider a sum of three profiles to fit the absorption feature in the C IV emission line. Two profiles are used for the $\mathrm{C}$ III line and one single profile is used to fit simultaneously the O III and Al II lines. All other BELs are fitted with one single profile. We then subtract the BELs and the continuum from the spectra. We consider the residuals as coming from the emission blends of Fe II and Fe III. Hence the averaged and normalized residuals over all epochs define our first iron pseudo-continuum template. We can then proceed iteratively by including this pseudo-continuum iron template in the fitting procedure and rerun it. This gives a better fitting of the emission lines and defines an improved iron pseudo-continuum template. After five of these iterations, typically the fitting does not change significantly anymore.
Figure 10 shows an example of the fitting decomposition described above.

\subsection{Results}

The light curves for the continuum and for the emission lines can be constructed from the above multi-component decomposition. We show in Fig. 11 an example of variation in the brightest quasar image, A, for the continuum and for two BELs. The error bars give the photon noise, integrated over the corresponding wavelength range. In the right panel of Fig. 11, we show the variability of the continuum in intensity, $f_{0}$, and in slope, $\alpha_{v}$, for the 4 quasar images. It is immediately clear that the continuum variations with the largest amplitude are observed in image A, between HJD = 3600 and 3900 days, and in image $\mathrm{B}$ between $\mathrm{HJD}=3300$ and 3500 days. These variations are accompanied by an increase in steepness, i.e. when a quasar image gets brighter, it also gets bluer. This is particularly obvious in Fig. 12, where $f_{0}$ and $\alpha_{v}$ are strongly correlated for images A and $\mathrm{B}$, indicating that significant microlensing events occured in these two images.

Inaccurate alignment of the quasar images in the slit of the spectrograph is a possible instrumental effect that can mimic microlensing changes in the spectral slope of the quasar images. Indeed, small clipping of one of the quasar images would lead to a stronger flux loss at the bluer wavelengths, hence producing a color gradient in the spectrum and a decrease in the measured value $\alpha_{v}$. We have checked all the "through-slit" images taken before each spectrum. Not only do these images show that 

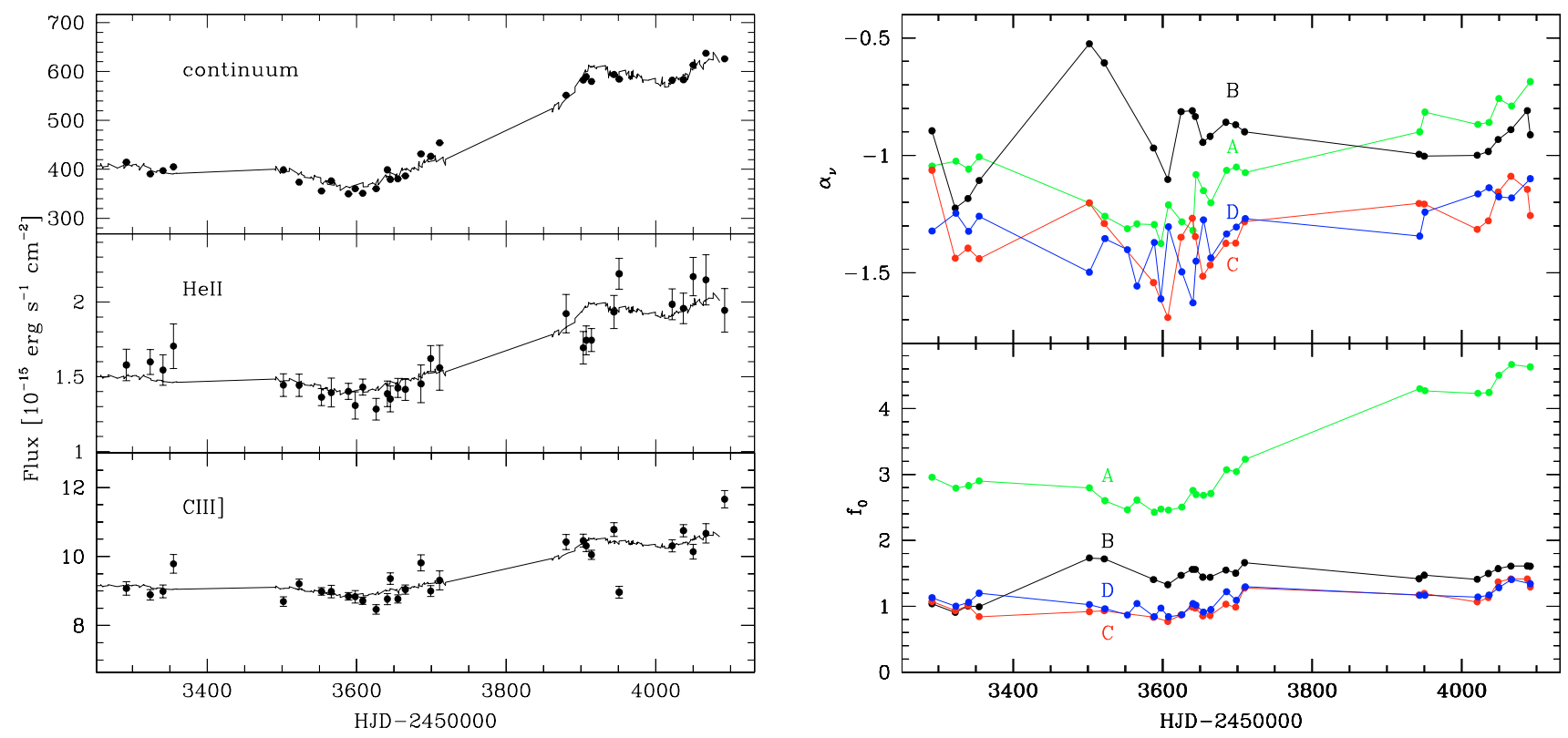

Fig. 11. Left: examples of light curves for the quasar image A. The integrated flux for the continuum, the He II, and C III BELs are given from top to bottom. The continuum is integrated over the entire available wavelength range. In each panel, we fit a scaled version (solid line) of the OGLE-III light curve (Udalski et al. 2006). This nicely illustrates that the BELs vary simultaneously and proportionally to the continuum. Right: variability of the best-fit parameters $\alpha_{v}$ and $f_{0}$ of the continuum (see Sect. 4.1). Note how the changes in slope of the continuum $\left(\alpha_{v}\right)$ follow those of the mean intensity $\left(f_{0}\right)$.

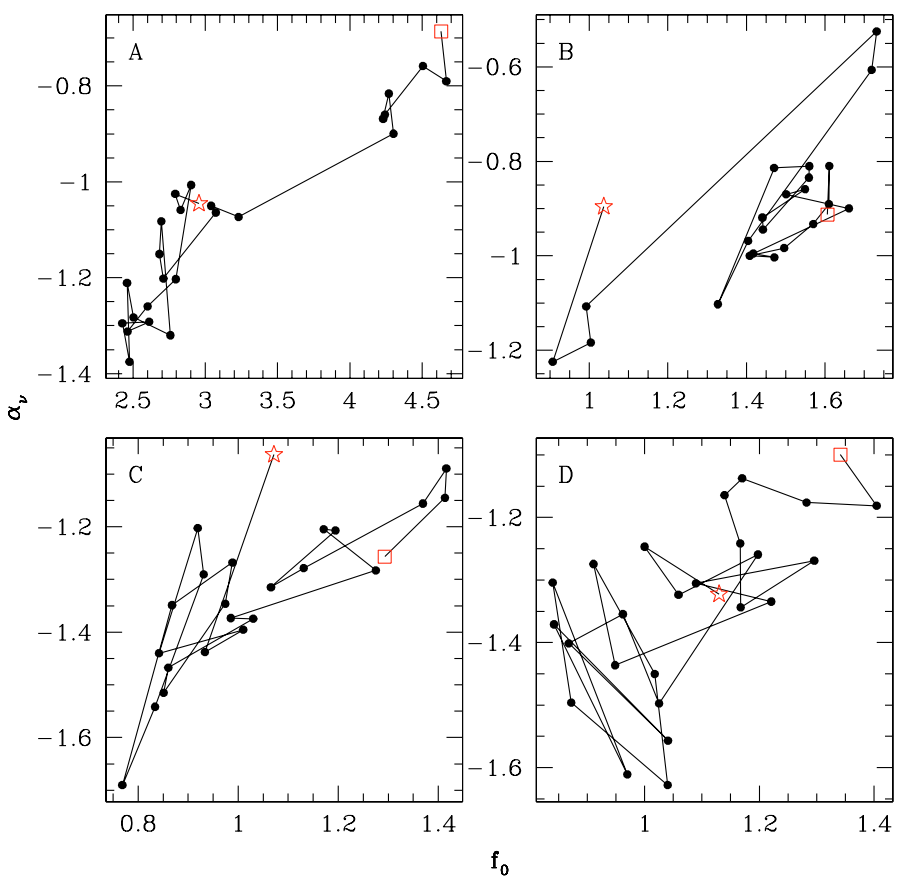

Fig. 12. Correlation between the intensity $f_{0}$ and slope $\alpha_{v}$ of the continuum spectra for all four quasar images. The points are connected chronologically. The first observation epoch is marked by a star and the last one by a square. The correlation is obvious in images A and B spanning a broad range of spectral slopes. In these two images, an increase in intensity is accompanied by an increase in steepness, i.e. when a quasar image gets brighter, it also gets bluer.

the alignment is correct, but it is also very easily reproducible from one epoch to another, even when the FORS1 has been dismounted from and remounted on the telescope.
We have also checked that our fitting procedure does not introduce any spurious correlation between $\alpha_{v}$ and $f_{0}$. We check this by using simulated spectra. In order to do that we take a reference spectrum for each quasar image and subtract its continuum. We then take random pairs of $\left(\alpha_{v}, f_{0}\right)$ parameters so that the $\alpha_{v}$ vs. $f_{0}$ plane is well sampled. We chose 400 such pairs and create the corresponding continuum to be added to the reference spectrum. The decomposition procedure is then run on the 400 spectra. We find no correlation at all between the measured $\alpha_{v}$ and $f_{0}$. In addition, the parameters used to build the simulated spectra are almost perfectly recovered by the decomposition procedure.

We conclude that genuine chromatic variations are present in the continuum of all images of QSO 2237+0305. The effect is most pronounced in image A during the last observing season, and in image $\mathrm{B}$ at the beginning of our monitoring. We show in the following that these observed variations are, in addition, well compatible with the OGLE-III single-band photometric observations.

\section{Microlensing variability in the OGLE-III photometry}

The photometric variations in most gravitationally-lensed quasars are dominated by the intrinsic variations of the quasar, typically of the order of $0.5-1.5 \mathrm{mag}$, hence making them useful to measure the time delays between the quasar images. Microlensing variations are usually smaller, in the range $0.05-0.1 \mathrm{mag}$ (e.g. the lensed quasars B $1600+434$ by Burud et al. 2000; RX J0911+0551 by Hjorth et al. 2002; SBS $1520+530$ by Burud et al. 2002b; FBQ $0951+2635$ by Jakobsson et al. 2005).

The Einstein Cross is different from this general behavior in two ways: (1) the time delays between each pair of images 


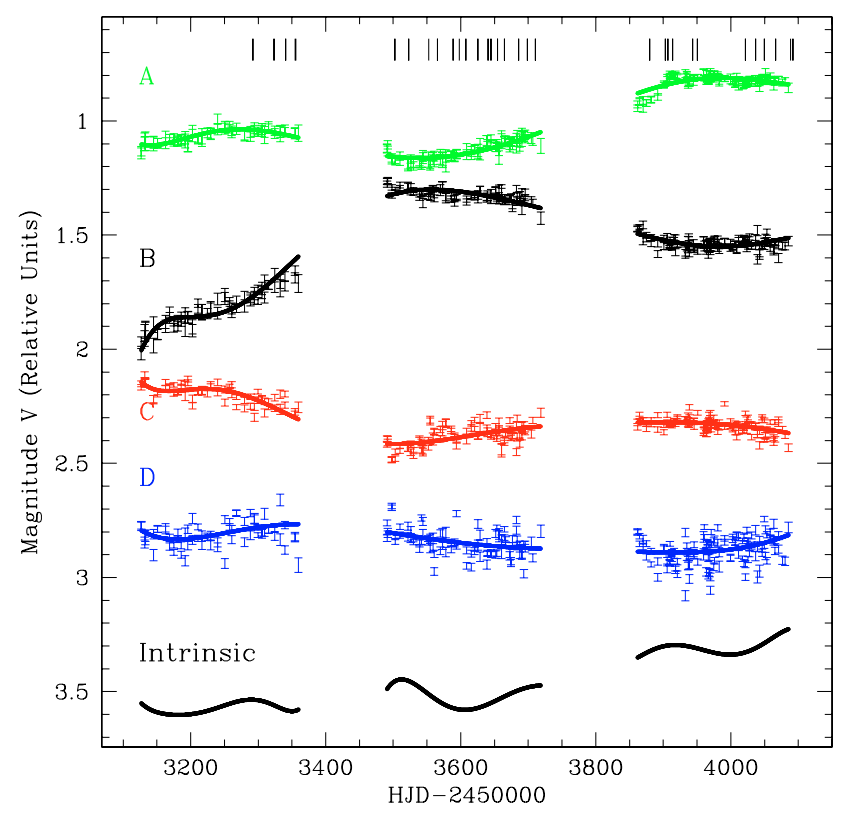

Fig. 13. Decomposition of the OGLE-III photometric light curves (Udalski et al. 2006) of the quasar images, into intrinsic quasar variations and microlensing-induced variations (see Sect. 5). The intrinsic variations are shown at the bottom of the figure as a continuous line, while the pure microlensing variations are the data points. The curves are shifted arbitrarily along the $y$-axis for clarity. The tickmarks at the top show the epochs of our observations.

are expected to be of the order of one day, hardly measurable; and (2) the microlensing variations dominate the light curves. For these two reasons, microlensing can be fairly well isolated in each quasar image, because it acts differently on the four sightlines.

To separate the intrinsic flux variations of the quasar from the microlensing ones, we perform a polynomial fit to the OGLE-III light curves (Udalski et al. 2006) of Fig. 9. This simple and fully analytical method has been developed by Kochanek et al. (2006), and is also described by Vuissoz et al. (2007). In the present application, the variations of each quasar image are modeled as a sum of two Legendre polynomials: one polynomial is common to all four quasar images and represents the intrinsic variations of the source while a second polynomial, different for each quasar image, represents the additional microlensing variations. In doing so, we rescale the OGLE-III error bars of each image by a factor equal to the flux ratio between each image and image C. This rescaling suppresses the potential problem existing if the fitting procedure considers the variation of image $\mathrm{A}$ (with the highest signal-to-noise) as the intrinsic variation of the quasar. The chosen order of the polynomial is 7 for the intrinsic variation, and 10 for the microlensing variation. Higher order polynomials do not significantly improve the fit. The results are displayed in Fig. 13, where the intrinsic variation of the source recovered by the simultaneous fit is shown together with the pure microlensing variations.

We check the efficiency of our method by generating artificial light curves and then using the above polynomial fit to recover the intrinsic and microlensing light curves. These artificial light curves are generated in the same way as described in Eigenbrod et al. (2005), and are composed of an intrinsic light curve to which we add microlensing fluctuations. Both are created in a random walk manner (i.e. not from polynomials). They are constructed to match the variability properties of the actual light curves, i.e. their timescale and amplitude of variation (for further details see Eigenbrod et al. 2005). We recover the simulated intrinsic light curve of the quasar with a typical error of less than $0.1 \mathrm{mag}$. The variations of more than $0.4 \mathrm{mag}$, shown in Fig. 13, both for microlensing and quasar variations, are well above the error estimated from the simulated light curves. In our simulations, we adopt the same photometric error bars as in the light curves of all quasar images, i.e. the re-scaling of the error bars described above in the real data is taken into account. If, on the contrary, we adopt error bars that follow the photon noise, the fitting procedure considers the highest signal-to-noise light curve as the intrinsic quasar light curve.

The light curve most affected by microlensing is that of image $\mathrm{B}$, with a peak-to-peak amplitude of more than 0.7 mag over 3 years. The other quasar images show microlensing-induced variations of up to $0.4 \mathrm{mag}$, with quasar image A having a sharp event during the last observing season. The intrinsic quasar light curve displays a variation of about $0.4 \mathrm{mag}$.

The polynomial decomposition of the light curves are compatible with the spectroscopic results. Quasar images A and B, which have the largest microlensing contribution in Fig. 13, respectively at HJD 3500 days and HJD 3900 days, also have a sharp rise in $\alpha_{v}$ at the same epochs.

\section{Microlensing variability in the spectra}

Chromatic variations of the continuum of images A and B of QSO 2237+0305 are clearly seen in our data. In addition, differential magnification of the continuum with respect to the BELs is also seen in all four quasar images. Such effects have already been observed by Lewis et al. (1998) and Wayth et al. (2005), but only for data over two epochs. Our VLT spectra allow us to follow the variations over two full years, provided the intrinsic variations of the quasar are removed.

\subsection{Continuum and BELs relative magnifications}

Since the time delays in QSO 2237+0305 are negligible, taking the ratio between the above quantities in pairs of quasar images cancels the intrinsic variations. Let $F(t)$ be the intrinsic source flux, and $M_{i}, \mu_{i}$ be the macro and microlensing-magnifications of quasar image $i$, respectively. The observed flux ratio between images $i$ and $j$ at time $t$ is then:

$R_{i j}(t)=\frac{\mu_{i}(t) M_{i} F(t)}{\mu_{j}(t) M_{j} F(t)} \mathrm{e}^{-\left(\tau_{i}-\tau_{j}\right)}=\frac{\mu_{i}(t) M_{i}}{\mu_{j}(t) M_{j}} \mathrm{e}^{-\left(\tau_{i}-\tau_{j}\right)}$

The extinction $\mathrm{e}^{-\tau_{i}}$ remains constant in time and is relatively similar in all four quasar images as we show in Sect. 6.3. Hence, it is not expected to strongly affect our results and we will neglect it in the following. The macromagnifications $M_{i}$ are best estimated in the mid-IR and radio domain (Falco et al. 1996; Agol et al. 2000). At these wavelengths, the source size is much larger than the typical spatial scale in the microcaustics network, hence leaving it fairly unaffected by microlensing (i.e. $\mu_{i}=1$ ). By multiplying $R_{i j}$ by $M_{j} / M_{i}$, using the mid-IR observations, we find the pure-microlensing magnification ratios:

$r_{i j}(t)=\frac{\mu_{i}(t)}{\mu_{j}(t)}$

In Fig. 14, we show the variations of $r_{i j}(t)$ for the integrated flux in the main emission lines and in the continuum. We plot in the 
A. Eigenbrod et al.: Microlensing variability in the Einstein Cross. I.
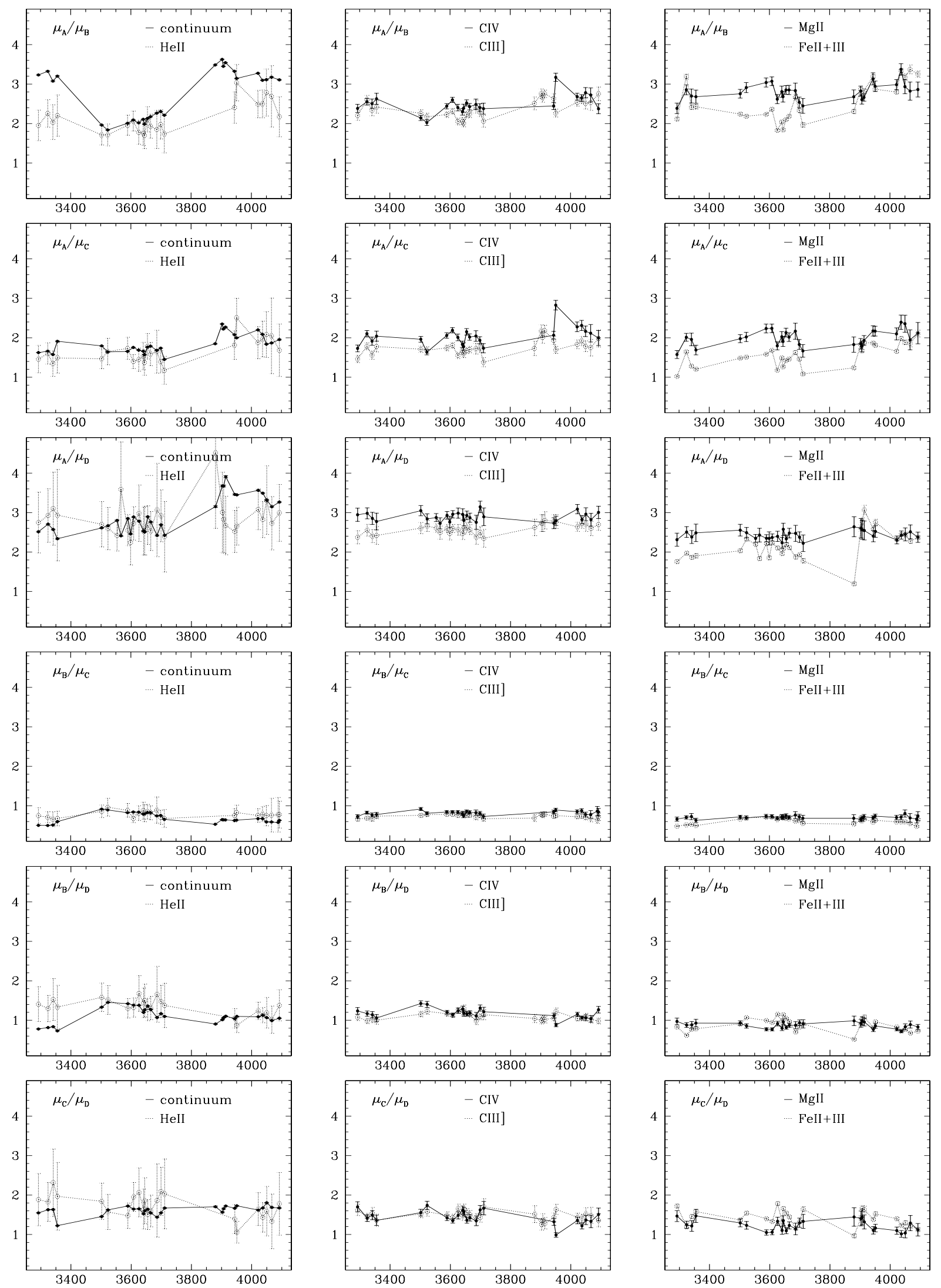

Fig. 14. Microlensing-magnification ratios $r_{i j}$ as a function of time (HJD-2450 000) for all possible combinations of image pairs. These ratios are corrected for the macromagnification by the lensing galaxy following Eq. (2). The ratios are given for the continuum, for the BELs He II, C IV, C III, Mg II and for the iron pseudo-continuum. 
figure all possible combinations of image ratios. Several interesting results can be drawn from this figure:

(a) With the exception of $r_{\mathrm{BD}}$ during the last season (i.e. HJD > 3900 days), none of the ratios $r_{i j}$ are close to 1, meaning that microlensing is acting at least in three quasar images during the entire monitoring. The evidence for $r_{\mathrm{BD}} \sim 1$ when HJD > 3900 days supports the absence of significant microlensing in images $\mathrm{B}$ and $\mathrm{D}$ during the last observing season.

(b) All the BELs have $r_{i j} \neq 1$ (except $r_{\mathrm{BD}}$ ). The $r_{i j}$ ratios in the BELs generally closely follow the value measured for the continuum. This demonstrates that the BLR is small enough - probably not larger than a few Einstein radii of a typical microlens in QSO 2237+0305- so that the BELs can be significantly affected. In addition, the variations observed in the BELs are correlated with those in the continuum.

(c) The largest changes of magnification ratios involve images A (for HJD 3900 days) and B (for HJD 3500 days). This is seen already in Fig. 13, but the ratios shown here allow us to make sure that the variations are only due to microlensing.

(d) The ratios $r_{\mathrm{A} j}$ and $r_{\mathrm{C} j}$ deviate significantly from 1 during the whole observing period. As an example, we measure in the continuum (resp. C III) $r_{\mathrm{AD}} \sim 2.6$ (resp. 2.8) for HJD $<3900$ days and $r_{\mathrm{CD}} \sim 1.6$ (resp. 1.6). Interestingly, these ratios have remained roughly the same since the beginning of the OGLE-III campaign (i.e. HJD 2000 days). Our values of $r_{\mathrm{AD}}$ and $r_{\mathrm{CD}}$ are also similar to the value measured in the $\mathrm{H} \beta$ emission line in August 2002 (HJD = 2496 days) by Metcalf et al. (2004). This strongly suggests that images $\mathrm{A}$ and $\mathrm{C}$ are affected by long-term micro/milli-lensing on periods longer than 5 years.

(e) The ratio $r_{\mathrm{CD}}$ is the most stable ratio along the monitoring campaign, indicating that no major (short) microlensing event occured in images $\mathrm{C}$ or D.

Because of (b) and (e), we can safely consider that during the time span of our observations, image $\mathrm{D}$ is the less affected by microlensing, both on short (i.e. of the order of a few weeks) and long timescales (years). This is consistent with the broadband microlensing light curves of Fig. 13.

We use image D as a reference to study the "short" microlensing events affecting image A at HJD 3900 days and image B at HJD 3500 days. During both events the emission lines are not magnified by the same amount (Fig. 14).

To quantify this, we compute in Table 2 the mean values of $r_{\mathrm{AD}}$ and $r_{\mathrm{BD}}$ during microlensing events and in more "quiescent" phases. For image A (i.e. $r_{\mathrm{AD}}$ ), we clearly see in this table that C IV, C III and He II show very similar magnification ratios, while the $\mathrm{Mg}$ II line is less magnified.

All the lines are less magnified than the continuum, consistent with a scheme where the continuum is emitted in the most compact region, and where other emission lines are emitted in larger regions, the largest region being the one with the lowest ionization potential (i.e. Mg II). Indeed the ionization potentials of the different lines are $47.9 \mathrm{eV}$ (C IV), $24.6 \mathrm{eV}$ (He II), $24.4 \mathrm{eV}$ (C III), and 7.6 eV (Mg II). The difference of magnification between C III and Mg II was not observed by Wayth et al. (2005). For image B, the same global trend is observed except that the relative errors on the $r_{\mathrm{BD}}$ ratios are higher due to the lower signal-to-noise ratio of the spectra of image $\mathrm{B}$. The effect is almost absent when $r_{\mathrm{AD}}$ and $r_{\mathrm{BD}}$ are computed in quiescent phases of components $\mathrm{A}$ and $\mathrm{B}$.

The behaviour of the Fe II+III emission is more difficult to interpret, as this complex is in fact a blend of many lines. However,
Table 2. Mean microlensing ratios for the continuum and for the main BELs. The mean values for $r_{\mathrm{AD}}=\mu_{\mathrm{A}} / \mu_{\mathrm{D}}$ and $r_{\mathrm{BD}}=\mu_{\mathrm{B}} / \mu_{\mathrm{D}}$ are computed for the observations around the epoch in the HJD line, i.e. during microlensing events or during quiescent phases. The values are given along with the dispersion of the points around the mean.

\begin{tabular}{lcccc}
\hline \hline & $\left\langle r_{\mathrm{AD}}\right\rangle$ & $\left\langle r_{\mathrm{AD}}\right\rangle$ & $\left\langle r_{\mathrm{BD}}\right\rangle$ & $\left\langle r_{\mathrm{BD}}\right\rangle$ \\
\hline HJD & $3900 \mathrm{~d}$ & $3500 \mathrm{~d}$ & $3500 \mathrm{~d}$ & $3300 \mathrm{~d}$ \\
\hline State & Micro-A & Quiet-A & Micro-B & Quiet-B \\
\hline Cont. & $3.46 \pm 0.24$ & $2.65 \pm 0.18$ & $1.28 \pm 0.12$ & $0.79 \pm 0.05$ \\
C IV & $2.89 \pm 0.13$ & $2.90 \pm 0.11$ & $1.22 \pm 0.11$ & $1.15 \pm 0.07$ \\
He II & $3.01 \pm 0.55$ & $2.70 \pm 0.33$ & $1.50 \pm 0.23$ & $1.39 \pm 0.10$ \\
C III & $2.71 \pm 0.07$ & $2.54 \pm 0.09$ & $1.17 \pm 0.09$ & $1.02 \pm 0.04$ \\
Mg II & $2.49 \pm 0.10$ & $2.40 \pm 0.10$ & $0.88 \pm 0.09$ & $0.91 \pm 0.04$ \\
Fe II+III & $2.56 \pm 0.23$ & $2.05 \pm 0.17$ & $0.99 \pm 0.15$ & $0.75 \pm 0.09$ \\
\hline
\end{tabular}

we note that the Fe II+III complex in image A is microlensed at about the same level as the $\mathrm{Mg}$ II line. In addition, the difference in magnification of the Fe II+III lines between a microlensing and a quiet phase is larger than for the other lines. This may suggest differential magnification of the emitting regions within the Fe II+III complex, i.e. that the Fe II+III is present both in compact and more extended regions, a conclusion also reached by Sluse et al. (2007).

\subsection{Line profiles}

We have discussed in the previous sections the global intensity changes in the emission lines of QSO $2237+0305$. We now investigate the possibility of a change in their profile. Such profile variations may be caused by differential magnification of regions with different velocities in the BLR. This can introduce, e.g., asymmetric changes or even peak displacement of the line (Lewis 1998; Abajas et al. 2002; Lewis \& Ibata 2004). Observational evidence for profile variations has been reported, e.g. in HE 2149-27 (Burud et al. 2002a); SDSS 1004+4112 (Richards et al. 2004; Gómez-Álvarez et al. 2006); SDSS J0924+0219 (Eigenbrod et al. 2006a); and RXJ 1131-1231 (Sluse et al. 2007).

We study the variability of the emission lines in the four quasar images using the continuum-subtracted spectra obtained from the decomposition procedure of Sect. 4.1. We concentrate on the C III emission line, which is easily decomposed into a sum of two components with full-width-half-maximum values about $1100 \mathrm{~km} \mathrm{~s}^{-1}$ and $5000 \mathrm{~km} \mathrm{~s}^{-1}$. We will refer to these components in the following as the "narrow" and "broad" components, although the difference made here between narrow and broad is only phenomenological. In particular, the narrow component is not associated, a priori, with the narrow line region (NLR).

We measure the microlensing magnification ratio $\mu_{i}$ (narrow) $/ \mu_{i}$ (broad) for the $\mathrm{C}$ III emission line in each quasar image. In image $\mathrm{A}$, the magnification of the broad component is $\sim 1.8$ times larger than the magnification of the narrow component, and this does not change drastically along our whole monitoring campaign. In the other quasar images, the microlensing-magnification of the narrow and broad components are comparable. This implies that the C III emission line is microlensed globally in all images, except in A. In the latter image, the broadest part of the line is more microlensed than the core, indicating that the broadest component of the $\mathrm{C}$ III emission is emitted in a more compact region than the core of the emission line. 

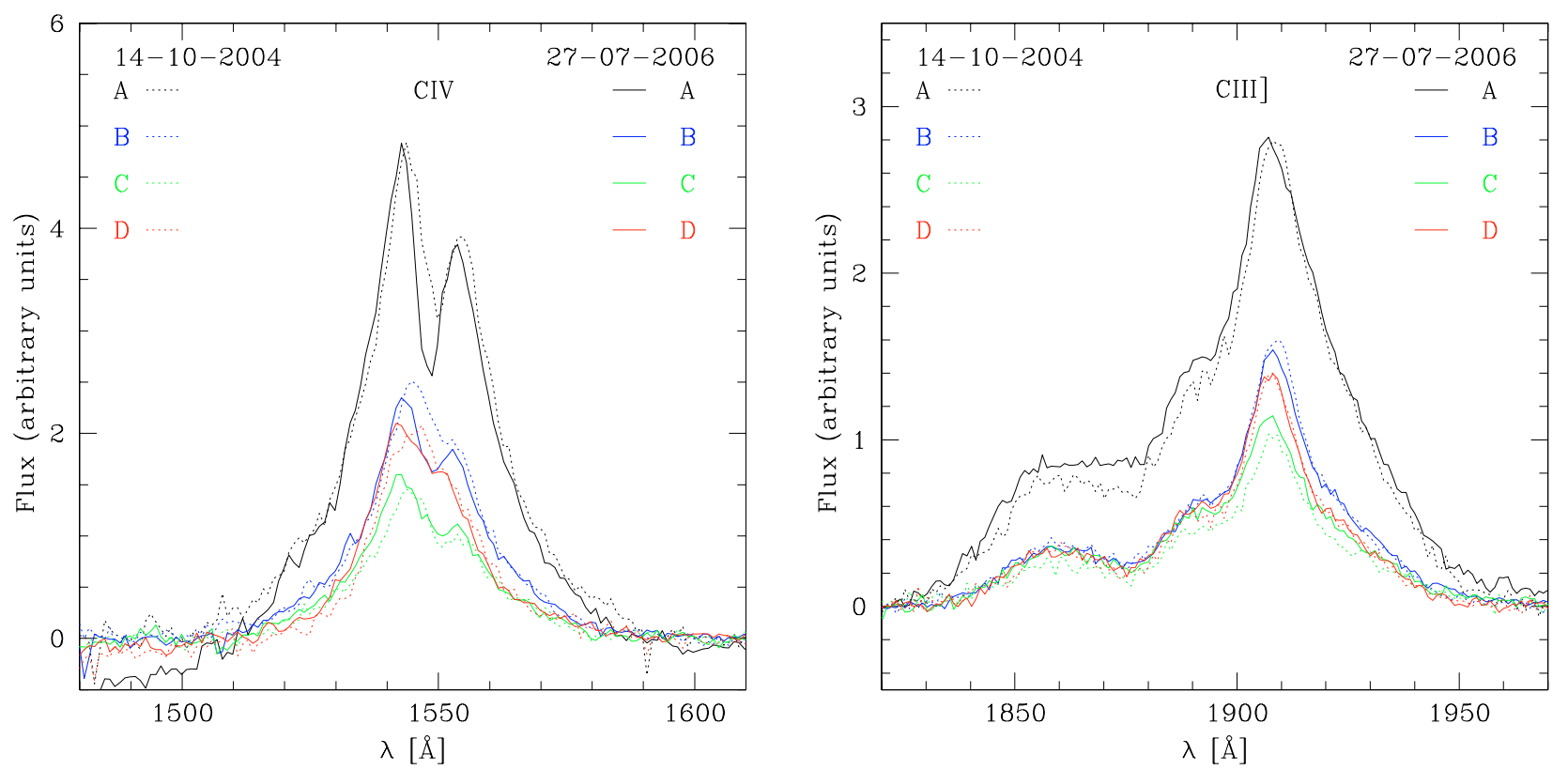

Fig. 15. The C IV (left) and C III (right) BEL profiles for quasar images A, B, C and D as observed at epoch \# 1 (14-10-2004, dotted) and epoch \# 24 (27-07-2006, solid). The first epoch is used as a reference while the second epoch falls within the high-magnification episode that occured at HJD 3900 days in image A (Sect. 6.2). In order to properly show the extra-magnification of the line wings during the strong microlensing episode of image A, we have multiplied the line profile in A observed on 14-10-2004 by a factor 1.05.

The core of the narrow C III emission line is probably associated mainly with photons emitted by the NLR: we observe nearly the same magnification ratio between the narrow and the broad components of the $\mathrm{C}$ III emission line than is reported between the narrow $\mathrm{O}$ III and the broad $\mathrm{H} \beta$ emission lines (Metcalf et al. 2004). This gives a hint that the narrow component of the $\mathrm{C}$ III emission line may be partly emitted by the NLR.

However, we do not measure the same microlensingmagnification ratios $r_{i j}$ in the narrow $\mathrm{C}$ III emission as those measured by Metcalf et al. (2004) in the narrow O III emission line. The $r_{\mathrm{BC}}, r_{\mathrm{BD}}, r_{\mathrm{CD}}$ ratios measured by these authors are similar for the $\mathrm{H} \beta \mathrm{BEL}$ and for the narrow $\mathrm{O}$ III emission line, but are different from 1 , which may be interpreted as a consequence of the microlensing of the NLR. If the NLR is indeed microlensed, the discrepancy between our values and the ones by Metcalf et al. (2004) can simply be explained by the fact that the amplitude of microlensing changes with time. However, there are also two other possible explanations. First, the size of the O III emission region might be comparable with the size of the macrocaustic of the lensing galaxy, such that the $\mathrm{O}$ III lensed images are extended and can be resolved by integral field spectroscopy (Metcalf et al. 2004; Yonehara 2006). This may lead to uncertainties in the flux measurements depending on the chosen size of the aperture. Finally, the discrepancy may be explained as well in terms of extinction by the lens (see Sect. 6.3).

The details of the C III profile also show variations. Our spectra show evidence for systematic broadening, by $\sim 10 \%$ of the $\mathrm{C}$ III emission line in quasar image A during our last observing season (HJD > 3900 days; Fig. 15). In addition, we note evidence for variations in the central parts of the C IV emission lines in all components and at most epochs. An example of these variations is shown in Fig. 15. Their interpretation will be much more complex than for the $\mathrm{C}$ III due to the decomposition of the line into three emission components plus an absorber-like feature.

To look for distortions in the line profiles of images B, C, and $\mathrm{D}$, we normalize the continuum-subtracted spectra of all images so that they share the same C III central intensity. The result is shown for selected epochs in Fig. 16. With this choice of normalization and in the absence of microlensing, the $\mathrm{C}$ III lines at a single epoch would match perfectly. It is conspicuous in Fig. 16 that this is not the case. First, we clearly see the effect of differential magnification between the core and the wings of image $\mathrm{A}$ at all epochs (the wings of C III in image A are always larger than in the other emission lines). The emission lines in $\mathrm{B}, \mathrm{C}$, and $\mathrm{D}$ are more similar to one another but there is no epoch where the three line profiles match perfectly, indicative that small microlensing fluctuations affect the $\mathrm{C}$ III line. Image $\mathrm{C}$ also shows line profile variations, even though the effect is less pronounced than in image A. Finally, we note the absence of any strong line profile variations of image B during the short-term microlensing event occuring in that image at HJD 3500 days.

\subsection{Differential extinction by the lensing galaxy}

Most of the magnification ratios $r_{i j}$ fluctuate around a mean value. The fluctuations themselves can only be explained by microlensing, but the value of the mean $\left\langle r_{i j}\right\rangle$ is usually different from 1 during our observations. This can, in principle, be explained either by long-term microlensing or by non variable extinction by dust in the lens.

In order to test the latter hypothesis, we use a simple and empirical diagnostic using ratio spectra of pairs of quasar images. In the absence of reddening and microlensing, these ratio spectra should be flat. If dust is present in different amounts on the lines of sight of the images, the ratio spectra will show a 

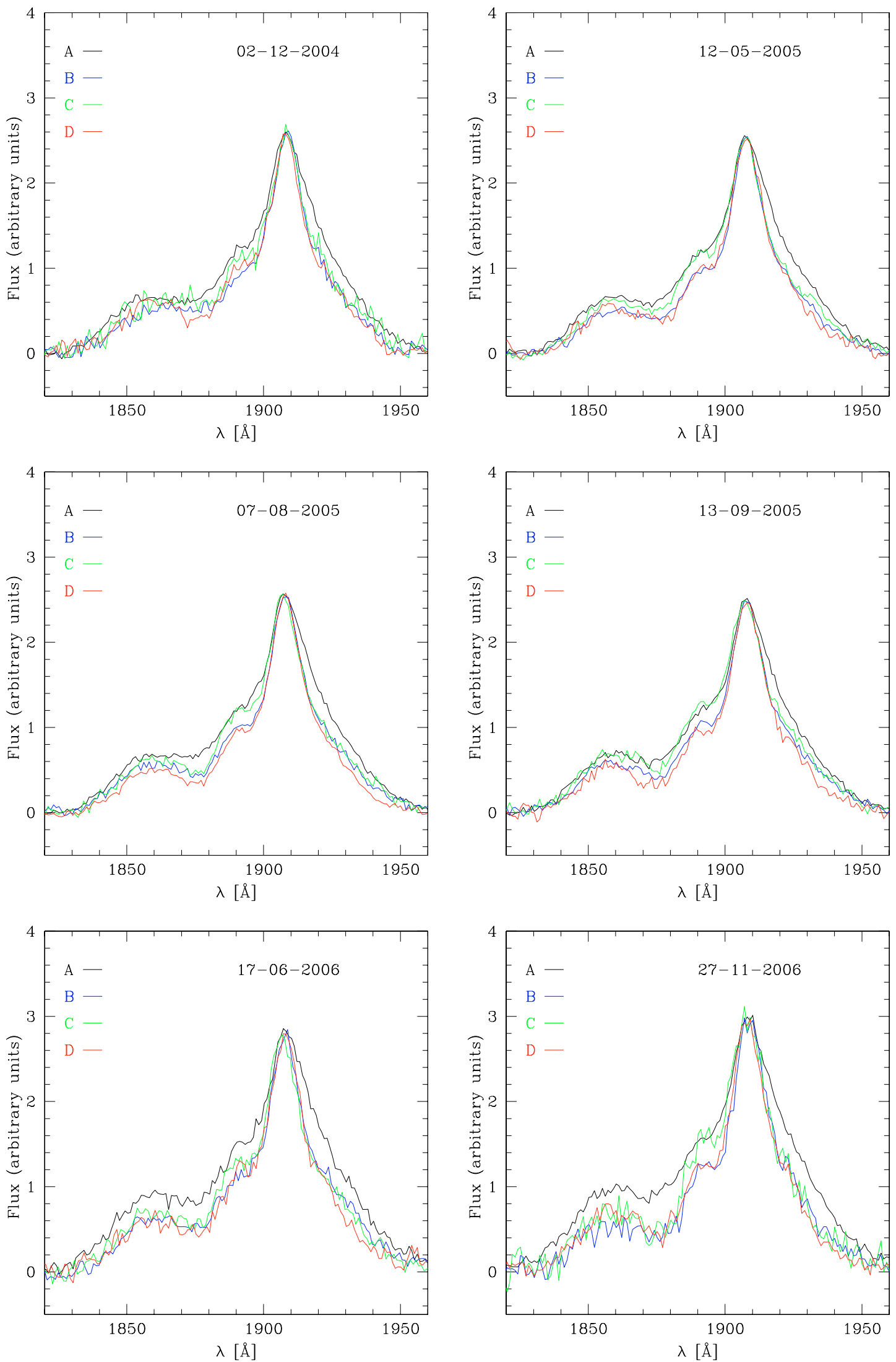

Fig. 16. Comparison of the C III BEL profiles of images A, B, C and D of the lensed quasar QSO $2237+0305$ on 6 different epochs. The line profiles are normalized so that they have the same peak value. This representation illustrates the difference in the line profiles of the 4 lensed images at several epochs. The most striking effect is the larger wings observed at all epochs in image A. These larger wings are caused by differential microlensing between the wings and the core of the $\mathrm{C}$ III line. Small microlensing induced fluctuations of the lines profiles are also observed in images B, C, and D. The epochs are chosen to sample the whole observing period. 
non-zero slope, constant with time. Any time-variable change of slope can safely be attributed to microlensing.

We find that the most useful pairs are the ones formed by $A \& C, B \& C$, and $C \& D$. Indeed, the $C / D$ ratio spectrum is found to be almost flat all along the years, indicating no significant differential extinction. This is not the case for the two other pairs of ratio spectra which show reddening of image $\mathrm{C}$ relative to both A and B, as also reported by Yee (1988). We estimate this differential reddening using the extinction law by Cardelli et al. (1989) and by assuming $R_{V}=3.1$. We find that the differential extinction $A_{V}(C)-A_{V}(A) \simeq A_{V}(C)-A_{V}(B)$ is in the range $0.1-0.3 \mathrm{mag}$. This range of values is sufficient to explain the discrepancy found between the $r_{i j}$ ratios measured in the C III narrow component and in O III of Sect. 6.2.

Finally, our estimates of the extinction in the lens are too small to explain the highest values of the mean magnification ratios observed in Fig. 14 and Table 2. For instance, the mean of the $r_{\mathrm{AB}}$ or $r_{\mathrm{AD}}$ ratio reaches values larger than 2. Static, longterm microlensing is therefore present in the Einstein Cross, at least in images $\mathrm{A}$ and $\mathrm{C}$.

\section{Conclusions}

This paper presents the first long-term (2.2 years) and wellsampled spectrophotometric monitoring of a gravitationally lensed quasar, namely the Einstein Cross QSO 2237+0305. The mean temporal sampling is of one observation every second week. The observations are carried out with the VLT in a novel way, using the spectra of PSF stars, both to deblend the quasar images from the lensing galaxy and to carry out a very accurate flux calibration. This paper, the first of a series, describes the observations and the techniques used to extract the scientific information from the data.

Detailed inverse ray-shooting simulations will be needed to infer quantitative information on the internal structure of the lensed quasar, and will be the topic of the future papers. The main observational facts that these simulations will need to take into account can be summarized as follows.

We find that all images of QSO 2237+0305 are affected by microlensing both on the long and short timescales. Comparison of the image flux ratios with mid-IR measurements reveals that quasar images $\mathrm{A}$ and $\mathrm{C}$ are both affected by long-term microlensing on a period longer than 5 years. This long-term microlensing affects both the continuum and the BELs.

Furthermore, in quasar image A, the broad component of the $\mathrm{C}$ III line is magnified by a factor 1.8 larger than the narrow component. On the contrary, the other quasar images have the same magnification in the narrow and broad components.

On the short timescales, i.e. several months, images A and $\mathrm{B}$ are the most affected by microlensing during our monitoring campaign. Image $\mathrm{C}$ and especially $\mathrm{D}$ are the most quiescent. Image A shows an important brightening episode at HJD $~ 3900$ days, and image B at HJD 3500 days. We show that the continuum of these two images becomes bluer as they get brighter, as expected from microlensing magnification of an accretion disk.

We also report microlensing-induced variations of the BELs, both in their integrated line intensities and in their profiles. In image $\mathrm{A}$, we find that the profile of the $\mathrm{C}$ III line is broadened during the brightening episode at HJD 3900 days. The $\mathrm{C}$ III line profile in image $\mathrm{C}$ seems also to be broadened at several epochs. Broadening of the BELs in image $\mathrm{B}$ is less obvious.

Variations in the BEL intensities are detected mainly in images $\mathrm{A}$ and $\mathrm{B}$. Our measurements suggest that higher ionization
BELs like C IV, C III, are more magnified than lower ionization lines like $\mathrm{Mg}$ II. This is compatible with reverberation mapping studies and a stratified structure of the BLR. There is marginal evidence that regions of different sizes are responsible for the $\mathrm{Fe}$ ii+iii emission.

Finally, we estimate the differential extinction between pairs of quasar images due to dust in the lensing galaxy to be in the range $0.1-0.3 \mathrm{mag}$, with images $\mathrm{C}$ and $\mathrm{D}$ being the most reddened. This amount of differential extinction is too small to explain the large microlensing-magnification ratios involving images A and C. Long-term microlensing, beyond the duration of our observations, is therefore present in these images.

The timescales of the microlensing variations in QSO 2237+0305 are such that each microlensing event lasts about one observing season (i.e. 8 months), with gaps of several months between events. This means that a relatively loose observing rate of 1 spectrum every 15 days is sufficient to sample the events well enough. In addition, the Einstein Cross is the lensed quasar with the fastest and sharpest microlensing events. It is therefore unique in the sense that only a few years of monitoring can truly constrain the quasar structure on parsec scales (Kochanek 2004). In addition, in the case of the Einstein Cross, the very different behaviours of the BELs and the continuum with respect to microlensing offer considerable hope to reconstruct the two types of regions independently, using ray-shooting simulations.

With two more years of data, we expect to map a total of up to half a dozen microlensing events in the four quasar images, hence providing a unique and useful data set for microlensing and quasar studies.

Acknowledgements. We are extremely grateful to all ESO staff for their excellent work. The observations presented in this article have involved a lot of efforts from the ESO staff operating FORS1, to ensure accurate and reproducible mask alignment, to keep the best possible temporal sampling, and to meet the requested seeing value. This project is partially supported by the Swiss National Science Foundation (SNSF).

\section{References}

Abajas, C., Mediavilla, E., Muñoz, J. A., et al. 2002, ApJ, 576, 640

Agol, E., \& Krolik, J. 1999, ApJ, 524, 49

Agol, E., Jones, B., \& Blaes, O. 2000, ApJ, 545, 657

Burud, I., Hjorth, J., Jaunsen, A. O., et al. 2000, ApJ, 544, 117

Burud, I., Courbin, F., Magain, P., et al. 2002a, A\&A, 383, 71

Burud, I., Hjorth, J., Courbin, F., et al. 2002b, A\&A, 391, 481

Cardelli, J. A., Clayton, G. C., \& Mathis, J. S. 1989, ApJ, 345, 245

Chartas, G., Eracleous, M., Agol, E., et al. 2004, ApJ, 606, 78

Colley, W. N., \& Schild, R. E. 2003, ApJ, 594, 97

Courbin, F., Magain, P., Kirkove, M., \& Sohy, S. 2000, ApJ, 529, 1136

Dietrich, M., Hamann, F., Appenzeller, I., et al. 2003, ApJ, 596, 817

Eigenbrod, A., Courbin, F., Vuissoz, C., et al. 2005, A\&A, 436, 25

Eigenbrod, A., Courbin, F., Dye, S., et al. 2006a, A\&A, 451, 747

Eigenbrod, A., Courbin, F., Meylan, G., et al. 2006b, A\&A, 451, 759

Falco, E. E., Lehar, J., Perley, R. A., et al. 1996, AJ, 112, 897

Filippenko, A. V. 1989, ApJ, 338, L49

Gómez-Álvarez, P., Mediavilla, E., Sánchez, S. F., et al. 2004, AN, 325, 132

Gómez-Álvarez, P., Mediavilla, E., Muñoz, J. A., et al. 2006, ApJ, 645, 5

Hjorth, J., Burud, I., Jaunsen, A. O., et al. 2002, ApJ, 572, 11

Huchra, J., Gorenstein, M., Kent, S., et al. 1985, AJ, 90, 691

Irwin, M. J., Webster, R. L., Hewett, P. C., et al. 1989, AJ, 98, 1989

Jakobsson, P., Hjorth, J., Burud, I., et al. 2005, A\&A, 431, 103

Kaspi, S., Smith, P. S., Netzer, H., et al. 2000, ApJ, 533, 631

Keeton, C. R., Burles, S., Schechter, P. L., et al. 2006, ApJ, 639, 1

Kent, S. M., \& Falco, E. E. 1988, AJ, 96, 1570

Kochanek, C. S. 2004, ApJ, 605, 58

Kochanek, C. S., Morgan, N. D., Falco, E. E., et al. 2006, ApJ, 640, 47

Koptelova, E., Shimanovskaya, E., Artamonov, B., et al. 2005, MNRAS, 356, 323

Lewis, G. F. 1998, MNRAS, 297, 69 
Lewis, G. F. \& Ibata, R. A. 2004, MNRAS, 348, 24

Lewis, G. F., Irwin, M. J., Hewett, P. C., et al. 1998, MNRAS, 295, 573

Magain, P., Courbin, F., \& Sohy, S. 1998, ApJ, 494, 452

Metcalf, R. B., Moustakas, L. A., Bunker, A. J., et al. 2004, ApJ, 607, 43

Mineshige, S., \& Yonehara, A. 1999, PASJ, 51, 497

Murray, N., Chiang, J., Grossman, S. A., \& Voit, G. M. 1995, ApJ, 451, 498

Nemiroff, R. J. 1988, ApJ, 335, 593

Press, W. H., Flannery, B. P., Teutolsky, S. A., \& Vetterling, W. T. 1986, in Numerical Recipes (Cambridge University Press)

Richards, G. T., Keeton, C. R., Pindor, B., et al. 2004, ApJ, 610, 679

Rix, H.-W., Schneider, D. P., \& Bahcall, J. N. 1992, ApJ, 104, 959

Schechter, P. L., Udalski, A., Szymański, M., et al. 2003, ApJ, 584, 657

Schneider, D. P., Turner, E. L., Gunn, J. E., et al. 1988, AJ, 95, 1619

Schneider, P., \& Wambsganss, J. 1990, AAP, 237, 42

Sluse, D., Claeskens, J.-F., Hutsemékers, D., et al. 2007, A\&A, 468, 885

Udalski, A., Szymański, M., Kubiak, M., et al. 2006, AcA, 56, 293 van Dokkum, P. G. 2001, PASP, 113, 1420

Vestergaard, M., \& Wilkes, B. J. 2001, ApJS, 134, 1

Vuissoz, C., Courbin, F., Sluse, D., et al. 2007, A\&A, 464, 845

Wambsganss, J., \& Paczyński, B. 1994, AJ, 108, 1156

Wambsganss, J., Paczyński, B., \& Schneider, P. 1990, ApJ, 358, 33

Wandel, A., Peterson, B. M. \& Malkan, M. A. 1999, ApJ, 526, 579

Wayth, R. B., O'Dowd, M., Webster, R. L. 2005, MNRAS, 359, 561

Wills, B. J., Netzer, H., \& Wills, D. 1985, ApJ, 288, 94

Wisotzki, L., Becker, T., Christensen, L., et al. 2003, A\&A, 408, 455

Wisotzki, L., Schechter, P. L., Chen, H.-W., et al. 2004, A\&A, 419, 31

Witt, H. J., Mao, S., \& Schechter, P. L. 1995, ApJ, 443, 18

Woźniak, P. R., Alard, C., Udalski, A., et al. 2000a, ApJ, 529, 88

Woźniak, P. R., Udalski, A., Szymański, M., et al. 2000b, ApJ, 540, L65

Yee, H. K. C. 1988, AJ, 95, 1331

Yonehara, A. 2006, ApJ, 646, 16 